MATHEMATICS OF COMPUTATION

Volume 00, Number 0, Pages 000-000

S $0025-5718(\mathrm{XX}) 0000-0$

\title{
CONTROL OF 2D SCALAR CONSERVATION LAWS IN THE PRESENCE OF SHOCKS
}

\author{
RODRIGO LECAROS ${ }^{1,3}$ AND ENRIQUE ZUAZUA ${ }^{1,2}$
}

\begin{abstract}
We analyze a model optimal control problem for a 2D scalar conservation law: The so-called inverse design problem, the goal being to identify the initial datum leading to a given final time configuration. The presence of shocks is an impediment for classical methods, based on linearization, to be directly applied. We develop an alternating descent method that exploits the generalized linearization that takes into account both the sensitivity of the shock location and of the smooth components of solutions. A numerical implementation is proposed using splitting and finite differences. The descent method we propose is of alternating nature and combines variations taking account of the shock location and those that take care of the smooth components of the solution. The efficiency of the method is illustrated by numerical experiments.
\end{abstract}

\section{INTRODUCTION}

There is an extensive literature on the control and inverse design of partial differential equations. When dealing with nonlinear models, most often, the analysis requires to linearize the system under consideration. This is why most of the existing results do not apply to hyperbolic conservation laws since the shock discontinuities of solutions are an impediment to linearize the system under consideration in a classical manner.

This paper is devoted to analyze this issue for $2 \mathrm{D}$ scalar conservation laws. To fix ideas we consider the problem of inverse design aiming to identify the initial datum so that the solution, at the final time, is close to a given final target.

To be more precise, given a finite time $T>0$, and a target function $u^{d} \in L^{2}\left(\mathbb{R}^{2}\right)$, we consider the functional $J$ to be minimized over a suitable class of initial data $\mathcal{U}_{a d}$, defined by

$$
J\left(u^{0}\right)=\frac{1}{2} \int_{\mathbb{R}^{2}}\left|\left(S(T) u^{0}\right)(x)-u^{d}(x)\right|^{2} d x
$$

Received by the editor May 2014.

2010 Mathematics Subject Classification. 35L67, 49J20, 90C31, 49M30, 35L65.

This work is supported by the Advanced Grants NUMERIWAVES/FP7-246775 of the European Research Council Executive Agency, FA9550-14-1-0214 of the EOARD-AFOSR, PI2010-04 and the BERC 2014-2017 program of the Basque Government, the MTM2011-29306-C02-00 and SEV-2013-0323 Grants of the MINECO.

The first author was partially supported by Basal-CMM project.

This work was done while the second author was visiting the CIMI (Centre International de Mathmatiques et Informatique) of Toulouse (France) and the University of Erlangen Nürnberg within the Humboldt Research Award program. 
where $S: L^{1}\left(\mathbb{R}^{2}\right) \cap L^{\infty}\left(\mathbb{R}^{2}\right) \cap B V\left(\mathbb{R}^{2}\right) \rightarrow L^{1}\left(\mathbb{R}^{2}\right) \cap L^{\infty}\left(\mathbb{R}^{2}\right) \cap B V\left(\mathbb{R}^{2}\right)$, is the semigroup

$$
S: u_{0} \rightarrow u=S u_{0}
$$

which associates to the initial condition $u_{0} \in L^{1}\left(\mathbb{R}^{2}\right) \cap L^{\infty}\left(\mathbb{R}^{2}\right) \cap B V\left(\mathbb{R}^{2}\right)$ the unique entropy solution $u: \mathbb{R}_{x}^{2} \times \mathbb{R}_{t} \rightarrow \mathbb{R}$ of the scalar conservation law

$$
\partial_{t} u+\operatorname{div}_{x} f(u)=0, \text { in } \mathbb{R}^{2} \times(0, T) ; \quad u(x, 0)=u^{0}(x), x \in \mathbb{R}^{2} .
$$

Here the flux $f: \mathbb{R} \rightarrow \mathbb{R}^{2}$ is assumed to be a smooth function: $f \in C^{1}\left(\mathbb{R}, \mathbb{R}^{2}\right)$. Thus, the problem under consideration reads: To find $u^{0, m i n} \in \mathcal{U}_{a d}$ such that

$$
J\left(u^{0, \min }\right)=\min _{u^{0} \in \mathcal{U}_{a b}} J\left(u^{0}\right) .
$$

The initial datum $u^{0}$ will be assumed to belong to a suitable class. But as a preliminary fact we remind that for $u^{0} \in L^{1}\left(\mathbb{R}^{2}\right) \cap L^{\infty}\left(\mathbb{R}^{2}\right) \cap B V\left(\mathbb{R}^{2}\right)$, there exists an unique entropy solution in the sense of Kružkov (see [28]) in the class $C^{0}\left([0, T] ; L^{1}\left(\mathbb{R}^{2}\right)\right) \cap L^{\infty}\left(\mathbb{R}^{2} \times[0, T]\right)$.

The inverse design problem under consideration is one of the classical optimization problems which is often addressed in the context of optimal aerodynamic design (see, for example, [21]).

As we will see, the existence of minimizers can be established under some natural assumptions on the class of admissible data $\mathcal{U}_{a d}$, using the well-posedness and compactness properties of solutions of the conservation law (1.2). The uniqueness of the minimizers is false, in general, due, in particular, to the possible presence of discontinuities in the solutions of (1.2).

Although this paper is devoted to this particular choice of $J$, that can also be handled by other methods, as, for instance, by backward resolution of the equation out of the final target, most of our analysis and numerical algorithms can be adapted to many other functionals and control problems, that would require to implement descent algorithms, which is the main content of the present paper. In particular our methods could allow to handle, for instance, the $2 \mathrm{D}$ version of the problems addressed in [27] and [14], where the control variable is the nonlinearity of the scalar conservation law. Our analysis can also be extended to higher space dimensions.

One of the classical approaches to effectively compute an accurate approximation of the minimizer, is constituted by the so-called continuous approach that consists in building a descent algorithm for the continuous optimization problem (1.3) to later discretise it. But this requires the computation of the Gâteaux derivative of $J$. However, when the constraint (1.2) involves a non smooth solution, this classical method does not apply. And this often occurs in the context of conservation laws where solutions may present shocks even for regular initial data $u^{0} \in C^{1}$ (see, for instance, L. C. Evans [18], Subsection 3.4.1, for details). When this occurs the formal linearization of the equation (1.2),

$$
\partial_{t} \delta u+\operatorname{div}_{x}\left(f^{\prime}(u) \delta u\right)=0,
$$

is not rigorously justified.

This problem was addressed in [12] in one space dimension for the inviscid Burgers' equation

$$
\partial_{t} u+\partial_{x}\left(\frac{u^{2}}{2}\right)=0, \text { in } \mathbb{R} \times(0, T) ; \quad u(x, 0)=u^{0}(x), x \in \mathbb{R},
$$


where the existence of minimizers was proved and an efficient descent algorithm based on a new optimization strategy was introduced, the so-called alternating descent direction method, which exploits the generalized gradient and linearization of the system.

Note that, when minimizing the functional (1.1), we are looking for an initial datum $u^{0}$ so that the solution of the conservation law reaches, or gets as close as possible to, the final target $u^{d}$. Thus, the problem can be viewed as a controllability one, the control being the initial datum. The problem can be recast in terms of identifying the set of reachable states at the final time $t=T$ for the semigroup generated by the conservation law. The later was addressed in [19] for parabolic problems and in [1] for 1D conservation laws. Much less is known in the context of hyperbolic conservation laws.

In $1 \mathrm{D}$, assuming the flux function is strictly convex, a characterization of the set of attainable states is given in [3] (see [2] for systems).

Furthermore, in [1], using Hopf and Lax-Olenik formula a method to obtain a solution of this optimal control problem was derived, by projecting the target $u^{d}$ into the set of states satisfying the well-known one-sided Lipschitz condition. This leads to iterative algorithms similar to the back-and-forth one developed in [4].

In practical applications, and in order to perform numerical computations and simulations, one has to replace the above continuous optimization problem and methods by discrete approximations. Then, it is natural to consider a discretization of system (1.2) and the functional $J$. If this is done in an appropriate way, the discrete optimization problem has minimizers, that are often taken, for small enough mesh-sizes, as approximations of the continuous minimizers. This is the so-called discrete or direct approach: "Discretize first and then optimize". There are however few results in the context of hyperbolic conservation laws proving rigorously the convergence of the discrete optimal controls towards the continuous ones, as the mesh-size goes to zero.

One of the main results of this paper ensures the $\Gamma$-convergence property of a numerical approximation of the inverse design problem based on a numerical approximation scheme for (1.1) and (1.2).

When optimal solutions have shock discontinuities, the purely discrete approach based on minimizing a discrete version of $J$ obtained by means of a discretization of the conservation law (1.2) produces highly oscillating minimizing sequences and the effective descent rate is very weak. As a remedy we introduce the $2 \mathrm{D}$ version of the alternating descent method. It uses a $2 \mathrm{D}$ extension of generalized tangent vectors (introduced in [10] and [9] for 1D problems, for instance), which enables to linearize (1.2) around discontinuous solutions. The alternating method distinguishes and alternates the descent directions that move the shock and those that perturb the profile of the solution away of it, producing overall, a very efficient and fast descent algorithm.

Thus, one of the main contributions of this paper consists on developing a careful sensitivity analysis of the continuous equation (1.2) and functional (1.1), paying special attention to the possible presence of shock discontinuities in solutions. This extends to the $2 \mathrm{D}$ case the $1 \mathrm{D}$ analysis based on generalized tangent vectors [10,9].

Once this is done we then develop the computational version of the alternated descent strategy, which requires important further developments to cope with the 
$2 \mathrm{D}$ case, integrating tools, in particular, from image processing. We finally prove its efficiency by several numerical experiments.

The rest of this paper is organized as follows. In Section 2 we formulate the inverse design problem more precisely and prove the existence of minimizers.

In Section 3 we introduce the discrete approximation of the continuous inverse design problem, prove the existence of minimizers and their $\Gamma$-convergence in the sense that discrete inverse designs converge to continuous ones as the mesh-size parameters tend to zero.

As we shall see, purely discrete approaches based on the minimization of the resulting discrete functionals by descent algorithms lead to very slow iterative processes. We thus need to introduce an alternated descent algorithm that takes into account the possible presence of shock discontinuities in solutions. For doing this the first step is to develop a careful sensitivity analysis. This is done in Section 4.

In Section 5 we present the alternating descent method which combines the advantages of both the discrete approach and the sensitivity analysis in the presence of shocks.

In Section 6 we explain how to implement two descent algorithms. The discrete approach consists mainly in applying a descent algorithm to the discrete version $J_{\Delta}$ of the functional $J$. And the alternating descent method, by the contrary, is a continuous method based on the analysis of the previous section.

Section 7 is devoted to present details of the numerical implementation. In Section 8 we present some numerical experiments illustrating the overall efficiency of the method. We conclude discussing some possible extensions of the results and methods presented in the paper.

\section{Existence of Minimizers}

In this section we prove that, under certain conditions on the set of admissible initial data $\mathcal{U}_{a d}$, there exists at least one minimizer of the functional $J$, given in (1.1). To do this, we consider the class of admissible initial data $\mathcal{U}_{a d}$ as:

$$
\mathcal{U}_{a d}=\left\{f \in L^{\infty}\left(\mathbb{R}^{2}\right) \cap B V\left(\mathbb{R}^{2}\right), \operatorname{supp}(f) \subset K,\|f\|_{L^{\infty}}+T V(f) \leq C\right\},
$$

where $K \subset \mathbb{R}^{2}$ is a given compact set and $C>0$ a given constant. Here $T V(f)$ represent the total variation of $f$ in $\mathbb{R}^{2}$.

Note, however, that the same theoretical results and descent strategies we shall develop here can be applied to a much wider class of admissible sets.

Theorem 2.1. Assume that $u^{d} \in L^{2}\left(\mathbb{R}^{2}\right)$. Let $\mathcal{U}_{a d}$ be defined in (2.1) and $f$ be a $C^{1}$ function. Then the minimization problem,

$$
\min _{u^{0} \in \mathcal{U}_{a b}} J\left(u^{0}\right)
$$

has at least one minimizer $u^{0, \text { min }} \in \mathcal{U}_{\text {ad }}$. Moreover, uniqueness is false in general.

Proof. Firstly, we prove the existence of a minimizer.

Let $\left\{u_{n}^{0}\right\}_{n \in \mathbb{N}} \subset \mathcal{U}_{a d}$ be a minimizing sequence of $J$.

In view of the definition of $\mathcal{U}_{a d}$, the minimizing sequence $\left\{u_{n}^{0}\right\}_{n \in \mathbb{N}}$ is necessarily bounded in $B V$. Thanks to the compactness of the embedding from $B V_{l o c}$ into $L_{l o c}^{1}$ we deduce that, up to the extraction of subsequences, $u_{n}^{0}$ weakly* converges to $u_{*}^{0}$ in $B V\left(\mathbb{R}^{2}\right) \cap L^{\infty}\left(\mathbb{R}^{2}\right)$ but, simultaneously, $u_{n}^{0} \rightarrow u_{*}^{0}$ strongly in $L_{\text {loc }}^{1}\left(\mathbb{R}^{2}\right)$. Actually, this strong convergence holds in $L_{l o c}^{p}\left(\mathbb{R}^{2}\right)$ for all $1 \leq p<+\infty$. 
We also have $u_{*}^{0} \in \mathcal{U}_{a d}$. Let $u_{n}(x, t)$ and $u_{*}(x, t)$ be the entropy solutions of (1.2) with initial data $u_{n}^{0}$ and $u_{*}^{0}$ respectively. We claim that

$$
u_{n}(\cdot, T) \rightarrow u_{*}(\cdot, T) \text {, strongly in } L^{2}\left(\mathbb{R}^{2}\right) .
$$

Assuming for the moment that the claim is true we deduce that

$$
\inf _{u^{0} \in \mathcal{U}_{a d}} J\left(u^{0}\right)=\lim _{n \rightarrow \infty} J\left(u_{n}^{0}\right)=J\left(u_{*}^{0}\right),
$$

and we conclude that $u_{*}^{0}$ is a minimizer of $J$.

Let us now prove (2.3). Taking the structure of $\mathcal{U}_{a d}$ into account and using the maximum principle and the finite velocity of propagation that entropy solutions satisfy, it is easy to see that the support of all solutions for all $t \in[0, T]$ is uniformly included in the same compact set of $\mathbb{R}^{2}$. The needed compactness property is then a consequence of the compactness of the embedding $B V_{l o c} \subset L_{l o c}^{1}$. Therefore, it is sufficient to prove convergence in $L_{l o c}^{2}$. This is obtained from the $L^{1}$-contraction property of the equation (we refer Kružkov, Theorem 4.1 in [22]):

$$
\left\|u_{n}(\cdot, t)-u_{*}(\cdot, t)\right\|_{L^{1}} \leq\left\|u_{n}^{0}-u_{*}^{0}\right\|_{L^{1}}, \quad \forall t \in[0, T],
$$

which guarantees a uniform convergence of $u_{n}$ to $u_{*}$ in $L^{1}$. Using the uniform bound in $L^{\infty}$ we obtain (2.3).

This completes the proof of the existence of minimizers.

The uniqueness of the minimizer is in general false for this type of optimization problems. In fact, there are examples of target functions $u^{d}$ for which there exist two different minimizers $u_{1}^{0}$ and $u_{2}^{0}$ such that the corresponding solutions $u_{j}, j=1,2$ satisfy $u_{j}(T)=u^{d}, j=1,2$ in such a way that the minimal value of $J$ vanishes. We refer to [12] in the proof of Theorem 2.1 for a example in $1 \mathrm{D}$ which is easy to extend to $2 \mathrm{D}$. Indeed, we can consider

$$
\begin{gathered}
u_{1}^{0}(x)=\left\{\begin{array}{ccc}
1 & x \in(-T, 0) \\
0 & \text { otherwise, }
\end{array}\right. \\
u^{d}=\left\{\begin{array}{cc}
\frac{x}{T}+1 & x \in(-T, 0) \\
1 & x \in(0, T / 2) \\
\frac{1}{2}-\frac{x}{T} & x \in(-T / 2, T / 2) \\
0 & \text { otherwise },
\end{array}\right.
\end{gathered}
$$

and we consider a $2 \mathrm{D}$ extension,

$$
\tilde{u}_{i}^{0}(x, y)=\left\{\begin{array}{cl}
u_{i}^{0}(x) & y \in[-1,1] \\
0 & \text { otherwise, }
\end{array} \quad \tilde{u}^{d}(x, y)=\left\{\begin{array}{cc}
u^{d}(x) & y \in[-1,1] \\
0 & \text { otherwise }
\end{array}\right.\right.
$$

Therefore, using the flux $f(z)=\left(z^{2} / 2,0\right)$, we obtain that $\tilde{u}_{1}^{0}$ and $\tilde{u}_{2}^{0}$ are both different minimizers (see Figure 1).

Here an in which follows we shall often use the notation $u^{T}$ for the value of the solution $u$ of the conservation law under consideration at the final time $t=T$ : $u^{T}(x)=u(x, T)$.

Remark 2.2. The above proof is quite general and can be adapted to other optimization problems with different functionals and admissible sets, and in particular to the functional

$$
J\left(u^{0}\right)=\frac{1}{2} \int_{0}^{T} \int_{\mathbb{R}^{2}}\left|u(x, t)-u^{d}(x, t)\right|^{2} d x d t
$$



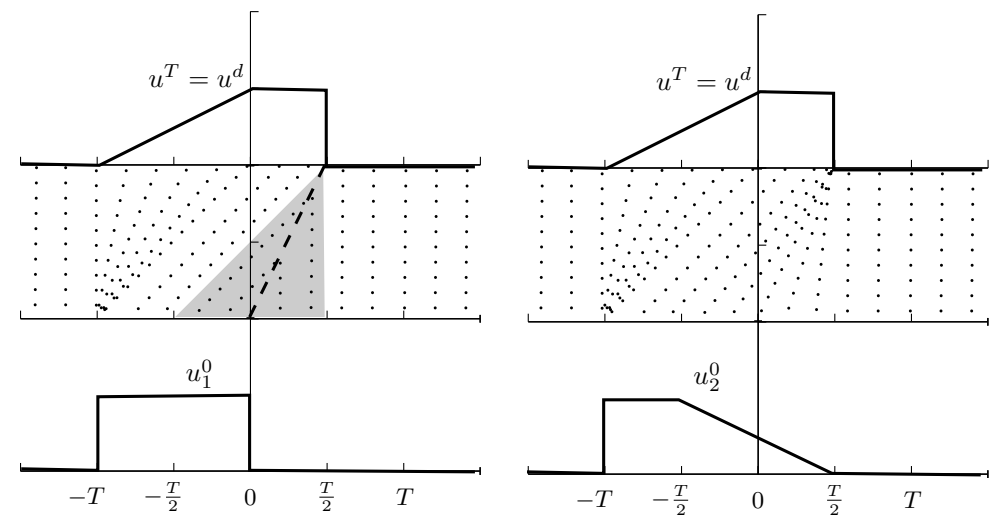

Figure 1. Plots of $u_{1}^{0}, u_{2}^{0}, u^{d}=u^{T}$ and the corresponding characteristic lines.

with $u^{d} \in L^{2}\left(\mathbb{R}^{2} \times(0, T)\right)$.

\section{The Discrete Minimization Problem}

The purpose of this section is to show that discrete minimizers obtained by a numerical approximation of (1.1) and (1.2), converge to a minimizer of the continuous problem as the mesh-size tends to zero. This justifies the usual engineering practice of replacing the continuous functional and model by discrete ones to compute an approximation of the continuous minimizer.

Let us introduce a mesh in $\mathbb{R}^{2} \times[0, T]$ given by $\left(x_{i}, y_{j}, t^{n}\right)=(i \Delta x, j \Delta y, n \Delta t)$ $(i=-\infty, \ldots, \infty ; j=-\infty, \ldots, \infty ; n=0, \ldots, N+1$, so that $(N+1) \Delta t=T)$, and let $u_{i, j}^{n}$ be a numerical approximation of $u\left(x_{i}, y_{j}, t^{n}\right)$ obtained as solution of a suitable discretization of the equation (1.2).

Let us consider the following approximation of the functional $J$ in (1.1):

$$
J_{\Delta}\left(u_{\Delta}^{0}\right)=\frac{\Delta x \Delta y}{2} \sum_{i=-\infty}^{\infty} \sum_{j=-\infty}^{\infty}\left(u_{i, j}^{N+1}-u_{i, j}^{d}\right)^{2},
$$

where $u_{\Delta}^{0}=\left\{u_{i, j}^{0}\right\}$ is the discrete initial datum and $u_{\Delta}^{d}=\left\{u_{i, j}^{d}\right\}=\Pi_{\Delta} u^{d}$ is the discretization of the target $u^{d}$ at $x_{i}, y_{j}$, respectively and $\Pi_{\Delta}$ is a discretization operator. A common choice consists in taking

$$
\Pi_{\Delta} u^{d}=u_{i, j}^{d}=\frac{1}{\Delta x \Delta y} \int_{x_{i-1 / 2}}^{x_{i+1 / 2}} \int_{y_{j-1 / 2}}^{y_{j+1 / 2}} u^{d}(x, y) d y d x,
$$

where $x_{i \pm 1 / 2}=x_{i} \pm \Delta x / 2$ and $y_{j \pm 1 / 2}=y_{j} \pm \Delta y / 2$.

Moreover, we introduce an approximation of the class of admissible initial data $\mathcal{U}_{a d}$ denoted by $\mathcal{U}_{a d, \Delta}$ and constituted by sequences $\varphi_{\Delta}=\left\{\varphi_{i, j}\right\}_{i, j \in \mathbb{Z}}$ for which the associated piecewise constant interpolation function, that we still denote by $\varphi_{\Delta}$, defined by

$$
\varphi_{\Delta}(x)=\varphi_{i, j}, \quad x \in\left(x_{i-1 / 2}, x_{i+1 / 2}\right), y \in\left(y_{j-1 / 2}, y_{j+1 / 2}\right),
$$


satisfies $\varphi_{\Delta} \in \mathcal{U}_{a d}$. Obviously, $\mathcal{U}_{a d, \Delta}$ coincides with the class of discrete vectors with support on those indices $i, j$ such that $x_{i}, y_{j} \in K$ and for which the discrete $L^{\infty}-$ norm and $T V$ are bounded above by the same constant $C$.

Let us consider $S_{\Delta}: l^{1}\left(\mathbb{Z}^{2}\right) \rightarrow l^{1}\left(\mathbb{Z}^{2}\right)$, an explicit numerical scheme for (1.2), where

$$
u_{\Delta}^{n}=S_{\Delta}^{n} u_{\Delta}^{0}
$$

is the approximation of the entropy solution $u(\cdot, t)=S(t) u^{0}$ of (1.2), i.e. $u_{\Delta}^{n} \simeq$ $S(t) u^{0}$, with $t=n \Delta t$. Here $S: L^{1}\left(\mathbb{R}^{2}\right) \cap L^{\infty}\left(\mathbb{R}^{2}\right) \cap B V\left(\mathbb{R}^{2}\right) \rightarrow L^{1}\left(\mathbb{R}^{2}\right) \cap L^{\infty}\left(\mathbb{R}^{2}\right) \cap$ $B V\left(\mathbb{R}^{2}\right)$, is the semigroup (solution operator of $(1.2)$ )

$$
S: u_{0} \rightarrow u=S u_{0},
$$

which associates to the initial condition $u_{0} \in L^{1}\left(\mathbb{R}^{2}\right) \cap L^{\infty}\left(\mathbb{R}^{2}\right) \cap B V\left(\mathbb{R}^{2}\right)$ the entropy solution $u$ of (1.2).

For each $\Delta=\Delta t$ (with $\lambda^{x}=\Delta t / \Delta x$ and $\lambda^{y}=\Delta t / \Delta y$ fixed, typically given by the corresponding CFL-condition for explicit schemes), it is easy to see that the discrete analogue of Theorem 2.1 holds. In fact this is automatic in the present setting since $\mathcal{U}_{a d, \Delta}$ only involves a finite number of mesh-points. But passing to the limit as $\Delta \rightarrow 0$ requires a more careful treatment. In fact, for that to be done, one needs to assume that the scheme under consideration (3.3), is a contraction in $l^{1}\left(\mathbb{Z}^{2}\right)$, satisfying a discrete version of (2.4). that

Thus, we consider the following discrete minimization problem: Find $u_{\Delta}^{0, \min }$ such

$$
J_{\Delta}\left(u_{\Delta}^{0, m i n}\right)=\min _{u_{\Delta}^{0} \in \mathcal{U}_{a d, \Delta}} J_{\Delta}\left(u_{\Delta}^{0}\right) .
$$

The following holds

Theorem 3.1. Assume that $u_{\Delta}^{n}$ is obtained by a numerical scheme (3.3), which satisfies the following

- For a given $u^{0} \in \mathcal{U}_{a d}, u_{\Delta}^{n}=S_{\Delta}^{n} \Pi_{\Delta} u^{0}$ converges to $u(x, t)$, the entropy solution of (1.2). More precisely, if $n \Delta t=t$ and $T_{0}>0$ is any given number,

$$
\max _{0 \leq t \leq T_{0}}\left\|u_{\Delta}^{n}-u(\cdot, t)\right\|_{L^{1}} \rightarrow 0, \quad \text { as } \quad \Delta \rightarrow 0 .
$$

- The map $S_{\Delta}$ is $L^{\infty}$-stable, i.e.

- The map $S_{\Delta}$ is a contraction in $l^{1}\left(\mathbb{Z}^{2}\right)$ i.e.

$$
\left\|S_{\Delta} u_{\Delta}^{0}-S_{\Delta} v_{\Delta}^{0}\right\|_{L^{1}} \leq\left\|u_{\Delta}^{0}-v_{\Delta}^{0}\right\|_{L^{1}}
$$

Then:

- For all $\Delta$, the discrete minimization problem (3.4) has at least one solution $u_{\Delta}^{0, \min } \in \mathcal{U}_{a d, \Delta}$

- Any accumulation point of $u_{\Delta}^{0, m i n}$ with respect to the weak-* topology in $L^{\infty}$, as $\Delta \rightarrow 0$, is a minimizer of the continuous problem (2.2).

Proof. (of Theorem 3.1) The existence of discrete minimizers in the first statement of the theorem is obvious in this case since we are dealing with a finite-dimensional problem. Actually, at this point only the continuity property (3.7) of $S_{\Delta}$ is necessary. 
The second statement is less trivial. And the other properties are required to guarantee the compactness of the numerical solutions as $\Delta \rightarrow 0$.

Let us assume that the following property holds: If $u_{\Delta}^{0} \in \mathcal{U}_{a d, \Delta}$ satisfies $u_{\Delta}^{0} \rightarrow u_{*}^{0}$ in $L^{\infty}\left(\mathbb{R}^{2}\right)$ with respect to the weak-* topology, then

$$
S_{\Delta}^{n} u_{\Delta}^{0} \rightarrow S(t) u_{*}^{0}, \quad \text { strongly in } L^{2},
$$

for all $t=n \Delta t$. This is a discrete version of (2.3).

We now follow a standard $\Gamma$-convergence argument. Using (3.8) we obtain the following continuity property:

$$
J_{\Delta}\left(u_{\Delta}^{0}\right) \rightarrow J\left(u^{0}\right) .
$$

Now, let $\hat{u}^{0} \in \mathcal{U}_{a d}$ be an accumulation point of $u_{\Delta}^{0, m i n}$ with respect to the weak-* topology of $L^{\infty}$. To simplify the notation we still denote by $u_{\Delta}^{0, \min }$ the subsequence for which $u_{\Delta}^{0, \text { min }} \rightarrow \hat{u}^{0}$, weakly-* in $L^{\infty}\left(\mathbb{R}^{2}\right)$, as $\Delta \rightarrow 0$. Let $v^{0} \in \mathcal{U}_{a d}$ be any other function. We are going to prove that

$$
J\left(\hat{u}^{0}\right) \leq J\left(v^{0}\right) .
$$

To do this we construct a sequence $v_{\Delta}^{0} \in \mathcal{U}_{a d, \Delta}$ such that $v_{\Delta}^{0} \rightarrow v^{0}$, in $L^{1}\left(\mathbb{R}^{2}\right)$, as $\Delta \rightarrow 0$ (we can consider in particular the approximation $\Pi_{\Delta} v^{0}$ in (3.2)). Taking the continuity property (3.9) into account, we have

$$
J\left(v^{0}\right)=\lim _{\Delta \rightarrow 0} J_{\Delta}\left(v_{\Delta}^{0}\right) \geq \lim _{\Delta \rightarrow 0} J_{\Delta}\left(u_{\Delta}^{0, \min }\right)=J\left(\hat{u}^{0}\right),
$$

which proves (3.10).

Finally we need to prove (3.8). The class of initial data $\mathcal{U}_{a d, \Delta}$ we are considering guarantees uniform local $B V$ bounds on the discrete solutions. This implies local compactness in $L^{1}$ and using that the supports are in the same compact set we obtain that $u_{\Delta}^{0} \rightarrow u_{*}^{0}$ in $L^{1}\left(\mathbb{R}^{2}\right)$. Now, setting $t=n \Delta t$, we have

$$
\left\|S_{\Delta}^{n} u_{\Delta}^{0}-S(t) u_{*}^{0}\right\|_{L^{1}} \leq\left\|S_{\Delta}^{n} u_{\Delta}^{0}-S_{\Delta}^{n} \Pi_{\Delta} u_{*}^{0}\right\|_{L^{1}}+\left\|S_{\Delta}^{n} \Pi_{\Delta} u_{*}^{0}-S(t) u_{*}^{0}\right\|_{L^{1}} .
$$

Using the contraction property (3.7) in (3.11) and the convergence of the scheme under consideration (3.5), we obtain

$$
\max _{0 \leq t \leq T_{0}}\left\|S_{\Delta}^{n} u_{\Delta}^{0}-S(t) u_{*}^{0}\right\|_{L^{1}} \rightarrow 0, \quad \text { as } \quad \Delta \rightarrow 0 .
$$

We note that $\left\|S(t) u_{*}^{0}\right\|_{L^{\infty}} \leq\left\|u_{*}^{0}\right\|_{L^{\infty}}$ and using the $L^{\infty}$-stability property (3.6), we have

$$
\max _{0 \leq t \leq T_{0}}\left\|S_{\Delta}^{n} u_{\Delta}^{0}-S(t) u_{*}^{0}\right\|_{L^{2}}^{2} \leq C \max _{0 \leq t \leq T_{0}}\left\|S_{\Delta}^{n} u_{\Delta}^{0}-S(t) u_{*}^{0}\right\|_{L^{1}}
$$

where $C=\max _{0 \leq t \leq T_{0}}\left\|S_{\Delta}^{n} u_{\Delta}^{0}-S(t) u_{*}^{0}\right\|_{L^{\infty}(\mathbb{R})}$. Therefore, using (3.12) and (3.13) we obtain (3.8).

We need now to introduce a numerical approximation scheme for (1.2). This can be done directly by introducing a $2 \mathrm{D}$ finite difference scheme. But here we shall rather employ a dimensional splitting method.

Let us then introduce one-dimensional nonlinear difference numerical approximation schemes, $H_{\Delta}^{x}$ and $H_{\Delta}^{y}$, where $v_{\Delta}^{n}=\left(H_{\Delta}^{x}\right)^{n} v_{\Delta}^{0}$, and $w_{\Delta}^{n}=\left(H_{\Delta}^{y}\right)^{n} w_{\Delta}^{0}$ are the numerical solutions at time $t=n \Delta t$ for the one-dimensional problems

$$
\begin{aligned}
v_{t}+\left(f_{1}(v)\right)_{x} & =0, & w_{t}+\left(f_{2}(w)\right)_{y} & =0, \\
v(x, y, 0) & =v^{0}(x, y) & w(x, y, 0) & =w^{0}(x, y),
\end{aligned}
$$


where $f_{1}$ and $f_{2}$ are the two components of the flux: $f(z)=\left(f_{1}(z), f_{2}(z)\right)$. For example, we can consider 3-point conservative numerical approximation schemes, where $H_{\Delta}^{x}$ and $H_{\Delta}^{y}$ are given by

$$
\begin{aligned}
\left(H_{\Delta}^{x} v_{\Delta}\right)_{i, j} & =v_{i, j}-\frac{\Delta t}{\Delta x}\left(g_{1}\left(v_{i+1, j}, v_{i, j}\right)-g_{1}\left(v_{i, j}, v_{i-1, j}\right)\right) \\
\left(H_{\Delta}^{y} w_{\Delta}\right)_{i, j} & =w_{i, j}-\frac{\Delta t}{\Delta y}\left(g_{2}\left(v_{i+1, j}, v_{i, j}\right)-g_{2}\left(v_{i, j}, v_{i-1, j}\right)\right)
\end{aligned}
$$

and $g_{1}, g_{2}$ are the numerical fluxes. Those schemes are consistent with the corresponding equation in (3.14) when $g_{1}(u, u)=f_{1}(u)$ and $g_{2}(u, u)=f_{2}(u)$.

When the functionals $H^{x}(u, v, w)=v-\lambda^{x}\left(g_{1}(u, v)-g_{1}(v, w)\right)$ and $H^{y}(u, v, w)=$ $v-\lambda^{y}\left(g_{2}(u, v)-g_{2}(v, w)\right)$ with $\lambda^{x}=\Delta t / \Delta x$ and $\lambda^{y}=\Delta t / \Delta y$, are monotone increasing with respect to each argument, the schemes are also monotone. Those are particularly useful schemes in one space dimension, since the discrete solutions obtained with them converge to weak entropy solutions of the continuous conservation law, as the discretization parameters tend to zero, under a suitable CFL condition (see Ref. [22], Chap. 3, Th. 4.2).

Thus, we can consider a numerical approximation scheme for (1.2) combining splitting and finite differences, leading to the numerical approximation

$$
u_{\Delta}^{n}=\left(H_{\Delta}^{y} H_{\Delta}^{x}\right)^{n} u_{\Delta}^{0} .
$$

The convergence result for this scheme was established in Theorem 2 of [15]. More precisely, for a given $u^{0} \in L^{1}\left(\mathbb{R}^{2}\right) \cap L^{\infty}\left(\mathbb{R}^{2}\right)$, when $\Delta \rightarrow 0$ with $\lambda^{x}, \lambda^{y}$ fixed (satisfying a suitable CFL condition), $u_{\Delta}^{n}$ converges to $u(x, t)$, the entropy solution of (1.2): If $n \Delta t=t$ and $T_{0}>0$ is fixed,

$$
\max _{0 \leq t \leq T_{0}}\left\|\left(H_{\Delta}^{y} H_{\Delta}^{x}\right)^{n} \Pi_{\Delta} u^{0}-S(t) u^{0}\right\|_{L^{1}} \rightarrow 0, \quad \text { as } \quad \Delta t \rightarrow 0,
$$

provided the schemes $H_{\Delta}^{x}$ and $H_{\Delta}^{y}$ used component-wise are monotone, of conservation form, consistent with the one-dimensional operators in (3.14), and have continuous numerical fluxes. Thus, the scheme (3.17) satisfies (3.5).

In addition to the convergence property in [15] it is important to underline that the numerical scheme obtained by splitting as above also fulfills the contraction property (3.7) and the $L^{\infty}$-stability property (3.6). Note that according to Crandall-Tartar Lemma (see Lemma 5.2 in [22]) this is so since each of the $1 \mathrm{D}$ schemes $H_{\Delta}^{x}$ and $H_{\Delta}^{y}$ employed map $l^{1}\left(\mathbb{Z}^{2}\right)$ into $l^{1}\left(\mathbb{Z}^{2}\right)$, being monotonic and conservative.

All this analysis and results apply to the classical Godunov, Lax-Friedrichs and Engquist-Osher schemes, the corresponding numerical fluxes being:

$$
\begin{aligned}
g_{1}^{G}(u, v) & =\left\{\begin{array}{cc}
\min _{w \in[u, v]} f_{1}(w), & \text { if } u \leq v, \\
\max _{w \in[u, v]} f_{1}(w), & \text { if } u \geq v,
\end{array}\right. \\
g_{1}^{L F}(u, v) & =\frac{\left(f_{1}(u)+f_{1}(v)\right)}{2}-\frac{(v-u)}{2 \lambda^{x}}, \\
g_{1}^{E O}(u, v) & =\frac{f_{1}(u)+f_{1}(v)-\int_{u}^{v}\left|f^{\prime}(\tau)\right| d \tau}{2} .
\end{aligned}
$$

See Chapter 3 in [22] for more details.

These 1D methods, combined with dimensional splitting, satisfy the conditions of Theorem 3.1. 
Remark 3.2. One may also consider the Strang-type splitting

$$
u_{\Delta}^{n}=\left(H_{\frac{\Delta}{2}}^{y} H_{\Delta}^{x} H_{\frac{\Delta}{2}}^{y}\right)^{n} u_{\Delta}^{0},
$$

which is second order accurate in time for sufficiently smooth solutions. We have employed (3.17) since it is simpler to be implemented and because, for conservation laws, the second order accuracy of the Strang-type splittings can be lost for oblique shocks. We refer to [15] for an example of the loss accuracy.

Remark 3.3. We could also use other schemes to approximate the solution of (1.2) in two space dimensions, such as the genuinely 2D numerical scheme introduced in $[8,24]$. These schemes satisfy the conditions of the Theorem 3.1.

\section{Sensitivity AnAlysis: the CONTINuOUs APPROACH}

We divide this section in three subsections. Specifically, in the first one we consider the case where the solution $u$ of (1.2) has no shocks. In the second and third subsections we analyze the sensitivity of the solution and the functional in the presence of a single shock located on a regular surface without boundary.

4.1. Sensitivity without shocks. In this subsection we give an expression for the sensitivity of the functional $J$ with respect to the initial datum based on a classical adjoint calculus for smooth solutions. First we present a formal calculus and then we show how to justify it when dealing with a classical smooth solution for (1.2).

Let $C_{0}^{1}\left(\mathbb{R}^{2}\right)$ be the set of $C^{1}$ functions with compact support and let $u^{0} \in C_{0}^{1}\left(\mathbb{R}^{2}\right)$ be a given initial datum for which there exists a classical solution $u(x, t)$ of $(1.2)$ that can be extended to a classical solution in $t \in[0, T+\tau]$ for some $\tau>0$. Note that this imposes some restrictions on $u^{0}$ other than being smooth.

Let $\delta u^{0} \in C_{0}^{1}\left(\mathbb{R}^{2}\right)$ be any possible variation of the initial datum $u_{0}$. Due to the finite speed of propagation, this perturbation will only affect the solution in a bounded set of $\mathbb{R}^{2} \times[0, T]$. This simplifies the argument below that applies in a much more general setting provided solutions are smooth enough.

Then for $\varepsilon>0$ sufficiently small, the solution $u^{\varepsilon}(x, t)$ corresponding to the initial datum $u^{\varepsilon, 0}(x)=u^{0}(x)+\varepsilon \delta u^{0}(x)$, is also a classical solution in $(x, t) \in \mathbb{R}^{2} \times(0, T)$ and $u^{\varepsilon} \in C^{1}\left(\mathbb{R}^{2} \times[0, T]\right)$ can be written as

$$
u^{\varepsilon}=u+\varepsilon \delta u+o(\varepsilon) \text {, with respect to the } C^{1} \text { topology, }
$$

where $\delta u$ is the solution of the linearized equation,

$$
\partial_{t} \delta u+\operatorname{div}_{x}\left(f^{\prime}(u) \delta u\right)=0, \text { in } \mathbb{R}^{2} \times(0, T) ; \quad \delta u(x, 0)=\delta u^{0}(x), x \in \mathbb{R}^{2} .
$$

Now, we introduce the adjoint system,

$$
-\partial_{t} p-f^{\prime}(u) \cdot \nabla_{x} p=0, \text { in } \mathbb{R}^{2} \times(0, T) ; \quad p(x, T)=p^{T}(x), x \in \mathbb{R}^{2},
$$

where $p^{T}(x)=u(x, T)-u^{d}(x)$. Thus, if we denote by $\delta J$ the Gâteaux derivative of $J$ at $u^{0}$ in the direction $\delta u^{0}$. We have

$$
\delta J\left(u^{0}\right)\left[\delta u^{0}\right]=\int_{\mathbb{R}^{2}} p(x, 0) \delta u^{0} d x .
$$

Therefore, a descent direction for the continuous functional $J$, once we have computed the adjoint state. We just take:

$$
\delta u^{0}=-p(x, 0) .
$$


Under the assumptions above on $u^{0}, u, \delta u$ and $p$ can be obtained from their data $u^{0}(x), \delta u^{0}(x)$ and $p^{T}(x)$ by using the characteristic curves associated to (1.2). For the sake of completeness we briefly explain this below.

The characteristic curves associated to (1.2) are defined by

$$
x^{\prime}(t)=f^{\prime}(u(x(t), t))=f^{\prime}\left(u^{0}\left(x^{0}\right)\right), \quad t \in(0, T) ; \quad x(0)=x^{0} \in \mathbb{R}^{2} .
$$

They are straight lines whose slopes depend on the initial data. As we are dealing with classical solutions, $u$ is constant along such curves and, by assumption, two different characteristic curves do not meet each other in $\mathbb{R}^{2} \times[0, T+\tau]$. This allows to define $u$ in $\mathbb{R}^{2} \times[0, T+\tau]$ in a unique way from the initial data.

For $\varepsilon>0$ sufficiently small, the solution $u^{\varepsilon}(x, t)$ corresponding to the initial datum $u^{\varepsilon, 0}(x)$, has similar characteristics to those of $u$. This allows guaranteeing that two different characteristic lines do not intersect for $0 \leq t \leq T$ if $\varepsilon>0$ is small enough. Note that $u^{\varepsilon}$ may possibly be discontinuous for $t \in(T, T+\tau]$ if $u^{0}$ generates a discontinuity at $t=T+\tau$ but this is irrelevant for the analysis in $[0, T]$ we are carrying out. Therefore $u^{\varepsilon}(x, t)$ is also a classical solution in $(x, t) \in \mathbb{R}^{2} \times[0, T]$ and it is easy to see that the solution $u^{\varepsilon}$ can be written as (4.1) where $\delta u$ satisfies (4.2). Thus, the solution $\delta u$ along a characteristic line can be obtained from $\delta u^{0}$, i.e.

$$
\delta u(x(t), t)=\delta u^{0}\left(x^{0}\right) \exp \left(-\int_{0}^{t} \operatorname{div}_{x}\left(f^{\prime}(u)\right)(x(s), s) d s\right) .
$$

Finally, the adjoint system (4.3) is also solved by characteristics. This yields the steepest descent direction in (4.5) for the continuous functional:

$$
p(x, 0)=u^{0}(x)-u^{d}\left(x+T f^{\prime}\left(u^{0}(x)\right)\right) .
$$

Remark 4.1. Note that for classical solutions the Gâteaux derivative of $J$ at $u^{0}$ is given by (4.4) and this provides an obvious descent direction for $J$ at $u^{0}$, given by (4.5). However this fact is not very useful in practice since, even when we initialize the iterative descent algorithm with a smooth $u^{0}$, we cannot guarantee that the solution remains classical along the iterative process.

4.2. Sensitivity of the state in the presence of shocks. Inspired in several results on the sensitivity of solutions of conservation laws in the presence of shocks in one-dimension (see $[10,5,6,7,33,23]$ ), we present an extension to the twodimensional case.

We focus on the particular case of solutions having a single surface of shock without boundary. For more details about the structure of discontinuities in two space dimensions see [35].

We introduce the following hypothesis:

Hypothesis 4.2. Assume that $u(x, t)$ is a weak entropy solution of (1.2) with a discontinuity along a regular surface, of class $C^{2}$,

$$
\Sigma=\{(\varphi(s, t), t) \mid(s, t) \in \mathbb{R} \times[0, T]\}=\cup_{t \in[0, T]} \Sigma^{t} \subset \mathbb{R}^{3},
$$

where $\Sigma^{t}=\{(\varphi(s, t), t), s \in \mathbb{R}\} \subset \mathbb{R}^{3}$, is the shock curve at time $t$ and $\varphi(\cdot, t)$ is a regular parametrization. The solution $u(x, t)$ is Lipschitz continuous outside $\Sigma$.

We call $\hat{s}$ the classical tangent vector to $\Sigma^{t}$, i.e.

$$
\hat{s}=\frac{\left(\partial_{s} \varphi, 0\right)}{h_{s}}, \text { on } \Sigma^{t},
$$


where $h_{s}=\left\|\partial_{s} \varphi\right\|$. We note that for all $t \in[0, T], \Sigma^{t}$ is a flat curve, i.e. $\hat{s} \cdot \hat{k}=0$, with $\hat{k}=(0,0,1)$. And we define the normal unit vector at the curve $\Sigma^{t}$ as

$$
\hat{\nu}=\hat{s} \times \hat{k} \text {, on } \Sigma \text {. }
$$

On the other hand, $u(x, t)$ satisfies the Rankine-Hugoniot condition on $\Sigma$

$$
[u]_{\Sigma^{t}} n_{\Sigma}^{t}+[f(u)]_{\Sigma^{t}} \cdot n_{\Sigma}^{x}=0, \text { on } \Sigma,
$$

where $n_{\Sigma}=\left(n_{\Sigma}^{x}, n_{\Sigma}^{t}\right) \in \mathbb{R}^{2} \times \mathbb{R}$ is the normal vector on the surface $\Sigma$ which satisfies

$$
\hat{\nu} \cdot n_{\Sigma}>0, \quad \text { on } \Sigma \text {. }
$$

This sets the direction of the normal vector $n_{\Sigma}$.

Here and in the sequel we use the following notation for the jump across a surface:

$$
[u]_{\Sigma^{t}}(y)=\lim _{\varepsilon \rightarrow 0} u((y, t)+\varepsilon \hat{\nu}(y, t))-u((y, t)-\varepsilon \hat{\nu}(y, t)), \quad \forall y \in \Sigma^{t} .
$$

In this form, the surface $\Sigma$ divides the space $\mathbb{R}^{2} \times(0, T)$ in two parts, to the left and right of $\Sigma$, respectively: $Q_{-}$and $Q_{+}$, where

$$
Q_{ \pm}=\left\{(x, t) \in Q_{ \pm}^{t} \times(0, T)\right\},
$$

with $\partial Q_{ \pm}^{t}=\Sigma^{t}$, the outward normal to $Q_{ \pm}^{t}$ being $\mp \hat{\nu}$ for all $t \in[0, T]$.

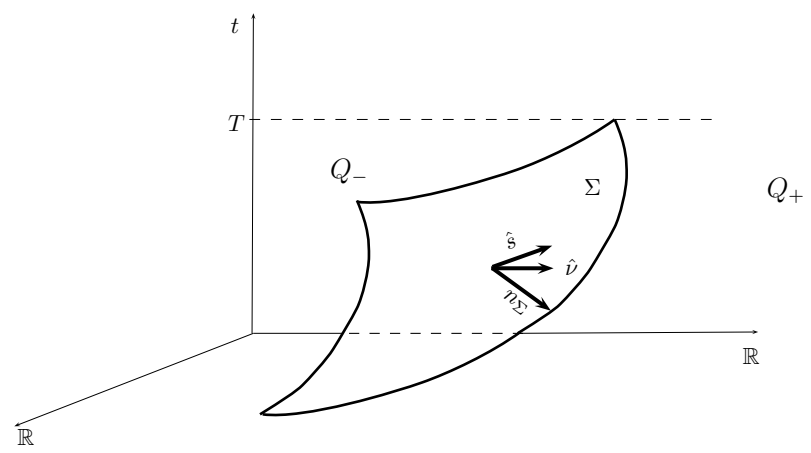

Figure 2. Scheme of the sets $Q_{-}, Q_{+}$and the shock surface $\Sigma$, with the vectors $n_{\Sigma}, \hat{\nu}, \hat{s}$ and $n_{\Sigma}^{x}$ in the space $\mathbb{R}^{2} \times[0, T]$.

As we will see, in the presence of shocks, to deal correctly with optimal control and design problems, the state of the system needs to be viewed as constituted by the pair $(u, \Sigma)$ combining the solution of $(1.2)$ and the shock location $\Sigma$. This is relevant in the analysis of sensitivity of solutions below and when applying descent algorithms.

Then the pair $(u, \Sigma)$ satisfies the system

$$
\begin{aligned}
\partial_{t} u+\operatorname{div}_{x}(f(u)) & =0, & & \text { in } Q_{-} \cup Q_{+} \\
{[u] n_{\Sigma}^{t}+[f(u)] n_{\Sigma}^{x} } & =0, & & \text { on } \Sigma \\
u(x, 0) & =u^{0}(x), & & x \in \mathbb{R}^{2} \backslash \Sigma^{0},
\end{aligned}
$$

where $\Sigma^{0}$ is the shock curve at $t=0$. In other words, the conservation law, that is fulfilled to both sides of the shock, is to be complemented with the RankineHugoniot condition. 
Let us now analyze the sensitivity of $(u, \Sigma)$ with respect to perturbations of the initial datum, in particular, with respect to variations $\delta u^{0}$ of the initial profile $u^{0}$ and $\delta \Sigma^{0}$ of the shock location $\Sigma^{0}$. To be more precise, we introduce a notion of generalized tangent vectors in $2 \mathrm{D}$.

Definition 4.3. Let $v: \mathbb{R}^{2} \rightarrow \mathbb{R}$ be a piecewise Lipschitz continuous function in $L^{1}\left(\mathbb{R}^{2}\right)$, with a single discontinuity on the curve $\sigma \subset \mathbb{R}^{2}$. We define $\mathcal{P}_{v}$ as the family of all continuous paths $\gamma:\left[0, \varepsilon_{0}\right] \rightarrow L^{1}\left(\mathbb{R}^{2}\right)$ with

(1) $\gamma(0)=v$ and $\varepsilon_{0}>0$ possibly depending on $\gamma$.

(2) For any $\varepsilon \in\left[0, \varepsilon_{0}\right]$ the functions $v^{\varepsilon}=\gamma(\varepsilon)$ are piecewise Lipschitz with a single discontinuity on the curve $\sigma^{\varepsilon}$ dividing the space $\mathbb{R}^{2}$ in two parts: $\Omega_{-}^{\varepsilon}$ and $\Omega_{+}^{\varepsilon}$, the sub-domains of $\mathbb{R}^{2}$ to the left and to the right of $\sigma^{\varepsilon}$ respectively,

$$
\Omega_{-}^{\varepsilon} \cup \sigma^{\varepsilon} \cup \Omega_{+}^{\varepsilon}=\mathbb{R}^{2} .
$$

(3) The curve $\sigma^{\varepsilon}$ depends continuously on $\varepsilon$ and there exists a constant $L$ independent of $\varepsilon \in\left[0, \varepsilon_{0}\right]$ such that

$$
\left|v^{\varepsilon}(x)-v^{\varepsilon}\left(x^{\prime}\right)\right| \leq L\left|x-x^{\prime}\right|,
$$

for all $x, x^{\prime}$ such that, $\sigma^{\varepsilon} \cap\left[x, x^{\prime}\right]=\emptyset$, when $\left[x, x^{\prime}\right]=\left\{\tau x^{\prime}+(1-\tau) x, \quad \tau \in\right.$ $[0,1]\}$.

Furthermore, we define the set $T_{v}$ of generalized tangent vectors of $v$ as the space of $(\delta v, \delta \varphi) \in L^{1}\left(\mathbb{R}^{2}\right) \times W^{1,1}(\sigma)$, for which the path $\gamma_{(\delta v, \delta \varphi)}$ given by

$$
\gamma_{(\delta v, \delta \varphi)}(\varepsilon)(x)=v(x)+\varepsilon \delta v(x)+\psi_{(\sigma, \delta \varphi)}^{\varepsilon}(x), \quad x \in \mathbb{R}^{2},
$$

where, in local coordinates i.e. $x=y+\lambda \delta \varphi(y) n_{\sigma}(y)$, for all $(y, \lambda) \in \sigma \times[0, \varepsilon)$, we have

$$
\psi_{(\sigma, \delta \varphi)}^{\varepsilon}(x)=\left\{\begin{array}{cc}
\operatorname{sgn}(\delta \varphi(y))[v]_{\sigma}(y), & (y, \lambda) \in \sigma \times[0, \varepsilon) \\
0, & \text { otherwise },
\end{array}\right.
$$

satisfies $\gamma_{(\delta v, \delta \varphi)} \in \mathcal{P}_{v}$.

Finally, we define the equivalence relation $\sim$ on $\mathcal{P}_{v}$ by

$$
\gamma \sim \gamma^{\prime} \text { if and only if } \lim _{\varepsilon \rightarrow 0} \frac{\left\|\gamma(\varepsilon)-\gamma^{\prime}(\varepsilon)\right\|_{L^{1}}}{\varepsilon}=0
$$

and we say that a path $\gamma \in \mathcal{P}_{v}$ generates the generalized tangent vector $(\delta v, \delta \varphi) \in$ $T_{v}$ if it is equivalent to $\gamma_{(\delta v, \delta \varphi)}$ as in (4.12).

Remark 4.4. The path $\gamma_{(\delta v, \delta \varphi)} \in \mathcal{P}_{v}$ in (4.12) represents, at first order, the variation of a function $v$ by adding a perturbation function $\varepsilon \delta v$ and by shifting the discontinuity by $\varepsilon \delta \varphi$. We note that $\operatorname{supp}\left(\psi_{(\sigma, \delta \varphi)}^{\varepsilon}\right) \subset\left[\sigma, \sigma^{\varepsilon}\right]$, meaning that the support is localized in the region between the original curve $\sigma$ and the new curve $\sigma^{\varepsilon}=\sigma+\varepsilon \delta \varphi n_{\sigma}$.

Let $u^{0}$ be the initial datum in (4.11) that we assume to be Lipschitz continuous to both sides of a single discontinuity located on the curve $\Sigma^{0}$, and consider a generalized tangent vector $\left(\delta u^{0}, \delta \varphi^{0}\right) \in C_{c}^{1}\left(\mathbb{R}^{2}\right) \times C_{c}^{1,1}\left(\Sigma^{0}\right)$. Let $u^{0, \varepsilon} \in$ $\mathcal{P}_{u^{0}}$ be a path which generates $\left(\delta u^{0}, \delta \varphi^{0}\right)$. For $\varepsilon$ sufficiently small the solution $u^{\varepsilon}(\cdot, t)$ of $(4.11)$ is Lipschitz continuous with a single discontinuity on the curve $\Sigma^{t, \varepsilon}$, for all $t \in[0, T]$. Therefore, $u^{\varepsilon}(\cdot, t)$ generates a generalized tangent vector 
$(\delta u(\cdot, t), \delta \varphi(\cdot, t)) \in L^{1}\left(\mathbb{R}^{2}\right) \times W^{1,1}\left(\Sigma^{t}\right)$. Thus, we note that the new position of the shock after perturbations of the form $\left(\delta u^{0}, \delta \varphi^{0}\right)$ is described by a function $\delta \varphi: \Sigma \rightarrow \mathbb{R}$ which denotes the variation of $\Sigma$ as follows,

$$
\Sigma^{\varepsilon}=\Sigma+\varepsilon \delta \Sigma=\{(y, t)+\varepsilon \delta \varphi \hat{\nu}, \quad(y, t) \in \Sigma\} .
$$

Then $\delta \varphi$ represents the displacement in the normal direction to $\Sigma^{t}$.

Now, let us introduce some definitions and notations: The divergence on the surfaces $\Sigma$ of the vector field $G$, i.e. $\operatorname{div}_{\Sigma}(G)$, is defined by

$$
\int_{\Sigma} \operatorname{div}_{\Sigma}(G) \psi d \Sigma=-\int_{\Sigma} \mathrm{G} \cdot \nabla_{\Sigma} \psi d \Sigma, \quad \forall \psi \in C_{c}^{1}\left(\mathbb{R}^{2} \times(0, T)\right),
$$

where $\nabla_{\Sigma}$ is tangential gradient (covariant derivative), defined as

$$
\nabla_{\Sigma} \psi=\nabla_{x, t} \psi-\left(\nabla_{x, t} \psi \cdot n_{\Sigma}\right) n_{\Sigma}, \quad \text { on } \Sigma .
$$

The following result describes the nature of the linearized system governing the dynamics of the generalized tangent vector $(\delta u, \delta \varphi)$.

Theorem 4.5. The equations for the new variables $\delta u$ and $\delta \varphi$ are

$$
\partial_{t} \delta u+\operatorname{div}_{x}\left(f^{\prime}(u) \delta u\right)=0, \quad \text { in } Q_{-} \cup Q_{+}
$$

$\operatorname{div}_{\Sigma}\left(\delta \varphi\left\|n_{\Sigma}^{x}\right\|\left([f(u)]_{\Sigma^{t}},[u]_{\Sigma^{t}}\right)\right)=\left(\left[f^{\prime}(u) \delta u\right]_{\Sigma^{t}},[\delta u]_{\Sigma^{t}}\right) \cdot n_{\Sigma}$, on $\Sigma$

$$
\begin{aligned}
\delta u(x, 0) & =\delta u^{0}(x), & & x \in \mathbb{R}^{2} \backslash \Sigma^{0} \\
\delta \varphi(x, 0) & =\delta \varphi^{0}(x), & & x \in \Sigma^{0} .
\end{aligned}
$$

Proof. (of Theorem 4.5) First let us define the function $G: \mathbb{R} \rightarrow \mathbb{R}^{3}$, as $G(z)=$ $(f(z), z)$. The system (4.11), including the Rankine-Hugoniot condition on the shock, can be rewritten in the following form

$$
\int_{\mathbb{R}^{2} \times(0, T)} G(u) \cdot \nabla_{x, t} \psi d x d t=0, \quad \forall \psi \in C_{c}^{1}\left(\mathbb{R}^{2} \times(0, T)\right) .
$$

Using Reynolds' transport theorem we obtain that the linearization of (4.20), is

$$
\begin{aligned}
\int_{Q_{-} \cup Q_{+}} & \delta u G^{\prime}(u) \cdot \nabla_{x, t} \psi d x d t \\
& -\int_{\Sigma} \delta \varphi\left(\nu \cdot n_{\Sigma}\right)[G(u)]_{\Sigma^{t}} \cdot \nabla_{x, t} \psi d \Sigma=0, \forall \psi \in C_{c}^{1}\left(\mathbb{R}^{2} \times(0, T)\right) .
\end{aligned}
$$

Considering $\psi$ with support outside $\Sigma$ in (4.21), we obtain

$$
\operatorname{div}_{x, t}\left(\delta u G^{\prime}(u)\right)=\partial_{t} \delta u+\operatorname{div}_{x}\left(f^{\prime}(u) \delta u\right)=0, \quad \text { in } Q_{-} \cup Q_{+} .
$$

On other hand, the Rankine-Hugoniot condition (4.8) can be rewritten as

$$
[G(u)]_{\Sigma^{t}} \cdot n_{\Sigma}=0, \text { on } \Sigma,
$$

and

$$
[G(u)]_{\Sigma^{t}} \cdot \nabla_{x, t} \psi=[G(u)]_{\Sigma^{t}} \cdot \nabla_{\Sigma} \psi, \quad \forall \psi \in C_{c}^{1}\left(\mathbb{R}^{2} \times(0, T)\right) .
$$

Using (4.22), (4.24) and (4.14) in (4.21), we have

$$
\begin{aligned}
0=-\int_{\Sigma} \psi & {\left[\delta u G^{\prime}(u)\right]_{\Sigma^{t}} \cdot n_{\Sigma} d \Sigma } \\
& +\int_{\Sigma} \operatorname{div}_{\Sigma}\left(\delta \varphi\left(\nu \cdot n_{\Sigma}\right)[G(u)]_{\Sigma^{t}}\right) \psi d \Sigma, \quad \forall \psi \in C_{c}^{1}\left(\mathbb{R}^{2} \times(0, T)\right) .
\end{aligned}
$$


Therefore, we obtain

$$
\operatorname{div}_{\Sigma}\left(\delta \varphi\left(\nu \cdot n_{\Sigma}\right)[G(u)]_{\Sigma^{t}}\right)=\left[\delta u G^{\prime}(u)\right]_{\Sigma^{t}} \cdot n_{\Sigma}, \quad \text { on } \Sigma .
$$

Thus, using the choice of the normal vector (4.9), we have $\left\|n_{\Sigma}^{x}\right\|=\hat{\nu} \cdot n_{\Sigma}$. Then, replacing this in (4.26), we obtain (4.17).

This concludes the proof.

Remark 4.6. The linearized system (4.16)-(4.19) is obtained, at least formally, by a perturbation argument. We introduce a perturbation of the data

$$
\left(u_{\varepsilon}^{0}, \Sigma_{\varepsilon}^{0}\right)=\left(u^{0}, \Sigma^{0}\right)+\varepsilon\left(\delta u^{0}, \delta \varphi^{0}\right),
$$

and compute the equations for the first order perturbation of the solution. This is in fact the usual approach in the study of the linearized stability of shocks. We refer to [23] for a detailed description of this method in the scalar case and [29] for more general systems of conservation laws in higher dimensions. This analysis leads to the formal expansion,

$$
\left(u_{\varepsilon}, \Sigma_{\varepsilon}\right)=(u, \Sigma)+\varepsilon(\delta u, \delta \varphi)+o(\varepsilon) .
$$

In $1 \mathrm{D}$, this expansion can be justified for general scalar one-dimensional conservation laws of the form,

$$
\partial_{t} u+\partial_{x}(f(u))=0
$$

when the flux $f \in C^{1}$ is convex in the context of duality solutions using the one sided Lipschitz condition (OSLC) (see [7]). This differentiability has been proved for more general situations, for instance in [10] for systems of conservation laws, or [33], for scalar equations in one-dimension but under more restrictive structural conditions on the solutions. In this paper we follow the later approach and our linearization is not justified in the general frame of Kružkov's entropy solutions.

4.3. The linearized transport equation on the shock surface $\Sigma$. The equation (4.17) and (4.19) on the function $\delta \varphi$ is a transport equation on the surface $\Sigma$. Here we explain how this equation can be solved.

Let us consider $\Sigma$ as in the Hypothesis 4.2 , and its parametrization $(\varphi(s, t), t)$. Then there exists $\varepsilon_{0}>0$, such that $H: \mathbb{R} \times(0, T) \times\left(-\varepsilon_{0}, \varepsilon_{0}\right) \rightarrow \mathbb{R}^{3}$ given by

$$
H(s, t, \lambda)=(\varphi(s, t), t)+\lambda n_{\Sigma}(s, t),
$$

is invertible, i.e. $H$ defines a local change of coordinates around of $\Sigma$, and the set $\Theta=H\left(\mathbb{R} \times(0, T) \times\left(-\varepsilon_{0}, \varepsilon_{0}\right)\right) \subset \mathbb{R}^{2} \times \mathbb{R}$ is a neighborhood of $\Sigma$. Thus, we can write the gradient of any function $\psi \in C^{1}(\Theta)$ in this local coordinates, and we obtain

$$
\nabla_{x, t} \psi=\frac{1}{\operatorname{det}(D H)}\left(\partial_{s} \tilde{\psi} \partial_{t} H \times \partial_{\lambda} H+\partial_{t} \tilde{\psi} \partial_{\lambda} H \times \partial_{s} H+\partial_{\lambda} \tilde{\psi} \partial_{s} H \times \partial_{t} H\right), \text { in } \Theta,
$$

where $\tilde{\psi}(s, t, \lambda)=\psi(H(s, t, \lambda))$ and $D H$ is the differential of $H$, i.e. $D H=$ $\left(\partial_{s} H, \partial_{t} H, \partial_{\lambda} H\right)$. Then, if we consider (4.28) on $\Sigma$, i.e. $\lambda=0$, the definition of tangent vector (4.7) and the normal vector $n_{\Sigma}$ in terms of the parametrization $H(s, t, 0)=(\varphi(s, t), t)$ of $\Sigma$, i.e.

$$
n_{\Sigma}=\frac{\partial_{s} H \times \partial_{t} H}{\left\|\partial_{s} H \times \partial_{t} H\right\|}=\frac{\hat{s} \times \partial_{t} H}{\left\|\hat{s} \times \partial_{t} H\right\|}=\left\|n_{\Sigma}^{x}\right\| \hat{\nu}+n_{\Sigma}^{t} \hat{k}, \quad \text { on } \Sigma,
$$


we obtain

$$
\nabla_{x, t} \psi=\frac{1}{h_{s}\left\|\hat{s} \times \partial_{t} H\right\|}\left(\partial_{s} \tilde{\psi} \partial_{t} H \times n_{\Sigma}+h_{s} \partial_{t} \tilde{\psi} n_{\Sigma} \times \hat{s}\right)+\partial_{\lambda} \tilde{\psi} n_{\Sigma}, \quad \text { on } \Sigma .
$$

With the notation $\left\|n_{\Sigma}^{x}\right\|=\frac{1}{\left\|\hat{s} \times \partial_{t} H\right\|}$ on $\Sigma$, we have

$$
\nabla_{\Sigma} \psi=\frac{\left\|n_{\Sigma}^{x}\right\|}{h_{s}}\left(\partial_{s} \tilde{\psi} \partial_{t} H \times n_{\Sigma}+h_{s} \partial_{t} \tilde{\psi} n_{\Sigma} \times \hat{s}\right) \text {, on } \Sigma .
$$

Thus, for any $F \in C^{1}(\Theta)$, using (4.29) and

$$
d \Sigma=\frac{h_{s}}{\left\|n_{\Sigma}^{x}\right\|} d s d t
$$

in the definition (4.14) we obtain

$$
\operatorname{div}_{\Sigma}(F)=\frac{\left\|n_{\Sigma}^{x}\right\|}{h_{s}}\left(\partial_{s}\left(F \cdot\left(\partial_{t} H \times n_{\Sigma}\right)\right)+\partial_{t}\left(h_{s} F \cdot\left(n_{\Sigma} \times \hat{s}\right)\right)\right) \text {, on } \Sigma .
$$

Let us denote $n^{\perp}=n_{\Sigma} \times \hat{s}$, which corresponds to the ninety degrees rotation of $n_{\Sigma}$ around $\hat{s}$. If $n_{\Sigma}=\left(n_{\Sigma}^{x}, n_{\Sigma}^{t}\right) \in \mathbb{R}^{2} \times \mathbb{R}$, we obtain

$$
n^{\perp}=\left(-\frac{n_{\Sigma}^{t}}{\left\|n_{\Sigma}^{x}\right\|} n_{\Sigma}^{x},\left\|n_{\Sigma}^{x}\right\|\right)
$$

On the other hand, we can consider $F$, such that $F_{n}=F \cdot n_{\Sigma}=0$, because by definition of tangent divergence (4.14), we have

$$
\int_{\Sigma} \operatorname{div}_{\Sigma}(F) \psi d \Sigma=\int_{\Sigma} \operatorname{div}_{\Sigma}(\tilde{F}) \psi d \Sigma, \quad \forall \psi \in C^{1}\left(\mathbb{R}^{2} \times(0, T)\right),
$$

where $\tilde{F}=F-\left(F \cdot n_{\Sigma}\right) n_{\Sigma}$ on $\Sigma$. Therefore, assuming

$$
F \cdot n_{\Sigma}=0, \quad \text { on } \Sigma,
$$

we obtain

$$
F \cdot n^{\perp}=\frac{1}{\left\|n_{\Sigma}^{x}\right\|} F \cdot \hat{k}, \quad \text { on } \Sigma,
$$

and an explicit computation gives

$$
\partial_{t} H \times n_{\Sigma}=\left(\left(\partial_{t} \varphi, 0\right) \cdot \hat{s}\right) n^{\perp}+\frac{1}{\left\|n_{\Sigma}^{x}\right\|} \hat{s}, \quad \text { on } \Sigma .
$$

Then, replacing (4.34) and (4.35) in (4.31), we obtain

$\operatorname{div}_{\Sigma}(F)=\frac{\left\|n_{\Sigma}^{x}\right\|}{h_{s}}\left(\partial_{s}\left(\frac{\left(\left(\partial_{t} \varphi, 0\right) \cdot \hat{s}\right)}{\left\|n_{\Sigma}^{x}\right\|} F \cdot \hat{k}+\frac{1}{\left\|n_{\Sigma}^{x}\right\|} F \cdot \hat{s}\right)+\partial_{t}\left(\frac{h_{s}}{\left\|n_{\Sigma}^{x}\right\|} F \cdot \hat{k}\right)\right)$, on $\Sigma$.

Now if we consider $F=\delta \varphi\left\|n_{\Sigma}^{x}\right\|\left([f(u)]_{\Sigma^{t}},[u]_{\Sigma^{t}}\right)$ on $\Sigma$, in (4.36), we have

$$
\operatorname{div}_{\Sigma}\left(\delta \varphi\left\|n_{\Sigma}^{x}\right\|\left([f(u)]_{\Sigma^{t}},[u]_{\Sigma^{t}}\right)\right)=\frac{\left\|n_{\Sigma}^{x}\right\|}{h_{s}}\left(\partial_{s}(B \delta \varphi)+\partial_{t}\left(h_{s}[u]_{\Sigma^{t}} \delta \varphi\right)\right) \text {, on } \Sigma,
$$

where the coefficient $B$ is given by $B=\left(\left(\partial_{t} \varphi, 0\right) \cdot \hat{s}\right)[u]_{\Sigma^{t}}+\left([f(u)]_{\Sigma^{t}}, 0\right) \cdot \hat{s}$. We note that the coefficient $h_{s}[u]_{\Sigma^{t}}$ does not vanish. Then the equation (4.17) with initial condition in $t=0$ is well-posed.

Thus using (4.37), we have that under the hypothesis (4.2), the coefficients in the equation (4.17) are Lipschitz continuous (i.e. $\left\|n_{\Sigma}^{x}\right\|[G(u)]_{\Sigma^{t}}$ is Lipschitz on $\Sigma$ ). The linearized system (4.16)-(4.19) has a unique solution which can be computed 
in two steps. The method of characteristics determines $\delta u$ in $Q^{-} \cup Q^{+}$, i.e. outside $\Sigma$, from the initial data $\delta u^{0}$, by the method of characteristics (note that system (4.16)-(4.19) has the same characteristics as (4.11)). This yields the value of $u$ and $\delta u$ to both sides of the shock $\Sigma$ and allows determining the coefficients and the right side of the equation that $\delta \varphi$ satisfies. Then, we solve this transport equation on $\Sigma$ to obtain $\delta \varphi$.

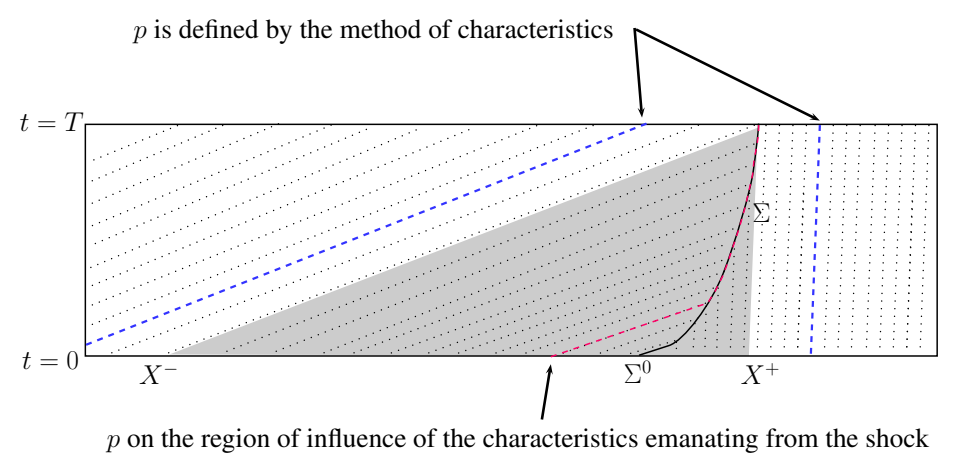

FiguRE 3. Characteristic lines entering on a shock and how they may be used to build the solution of the adjoint system both away from the shock and on its region of influence.

4.4. Sensitivity of $J$ in the presence of shocks. In this section we study the sensitivity of the functional $J$ with respect to variations associated with the generalized tangent vectors defined in the previous section. We first define an appropriate generalization of the Gâteaux derivative of $J$.

Definition 4.7. Let $J: L^{1}\left(\mathbb{R}^{2}\right) \rightarrow \mathbb{R}$ be a functional and $u^{0} \in L^{1}\left(\mathbb{R}^{2}\right)$ be Lipschitz continuous with a discontinuity along a regular curve $\Sigma^{0}$, of class $C^{2}$, an initial datum for which the solution of (1.2) satisfies hypothesis (4.2) and (4.13). $J$ is Gâteaux derivative differentiable at $u^{0}$ in a generalized sense if for any generalized tangent vector $\left(\delta u^{0}, \delta \varphi^{0}\right)$ and any family $u^{0, \varepsilon} \in \mathcal{P}_{u^{0}}$ associated to $\left(\delta u^{0}, \delta \varphi^{0}\right)$ the following limit exists,

$$
\delta J=\lim _{\varepsilon \rightarrow 0} \frac{J\left(u^{0, \varepsilon}\right)-J\left(u^{0}\right)}{\varepsilon},
$$

and it depends only on $\left(u^{0}, \Sigma^{0}\right)$ and $\left(\delta u^{0}, \delta \varphi^{0}\right)$, i.e. it does not depend on the particular family $u^{0, \varepsilon}$ which generates $\left(\delta u^{0}, \delta \varphi^{0}\right)$. The limit is the generalized Gâteaux derivative of $J$ in the direction $\left(\delta u^{0}, \delta \varphi^{0}\right)$.

The following result easily provides a characterization of the generalized Gâteaux derivative of $J$ in terms of the solution of the associated adjoint system (4.39), (4.40),(4.41), (4.42), (4.43) and (4.44).

Proposition 4.8. The Gâteaux derivative of $J$ can be written as follows

$$
\delta J\left(u^{0}\right)\left[\delta u^{0}, \delta \varphi^{0}\right]=\int_{\mathbb{R}^{2}} p(x, 0) \delta u^{0} d x-\int_{\Sigma^{0}} q(x, 0)[u]_{\Sigma^{0}} \delta \varphi^{0} d \sigma,
$$


where the adjoint state pair $(p, q)$ satisfies the system

$$
\begin{array}{rll}
\partial_{t} p+f^{\prime}(u) \cdot \nabla p & =0, & \text { in } Q_{-} \cup Q_{+} \\
{[p]_{\Sigma^{t}}} & =0, & \text { on } \Sigma \\
q(x, t) & =p(x, t), & (x, t) \in \Sigma \\
\left([f(u)]_{\Sigma^{t}},[u]_{\Sigma^{t}}\right) \cdot \nabla_{\Sigma} q & =0, & \text { on } \Sigma \\
p(x, T) & =u(x, T)-u^{d}(x), & x \in \mathbb{R}^{2} \backslash \Sigma^{T} \\
q(x, T) & =\frac{\left[\left(u(\cdot, T)-u^{d}\right)^{2} / 2\right]_{\Sigma^{T}}}{[u]_{\Sigma^{T}}}, & x \in \Sigma^{T} .
\end{array}
$$

Let us briefly comment the result of Proposition 4.8 before giving its proof. Formula (4.38) provides an obvious way to compute a first descent direction of $J$ at $u^{0}$. We just take

$$
\left(\delta u^{0}, \delta \varphi^{0}\right)=\left(-p(\cdot, 0), q(\cdot, 0)[u]_{\Sigma^{0}}\right) .
$$

Here, the value of $\delta \varphi^{0}$ must be interpreted as the optimal infinitesimal displacement of the discontinuity of $u^{0}$. However, it is important to underline that this $\left(\delta u^{0}, \delta \varphi^{0}\right)$ is not a generalized tangent vector in $T_{u^{0}}$ since $p(x, 0)$ is not continuous away from $\Sigma^{0}$. In fact in one dimension, $p(x, t)$ takes the constant value $q(T)$ in the whole triangular region occupied by the characteristics of (1.2) which meet the shock $\Sigma$. Thus, $p$ has, in general, two discontinuities at the boundary of this region and so will be for $p(x, 0)$ (see [12]) and the scheme in Figure 3.
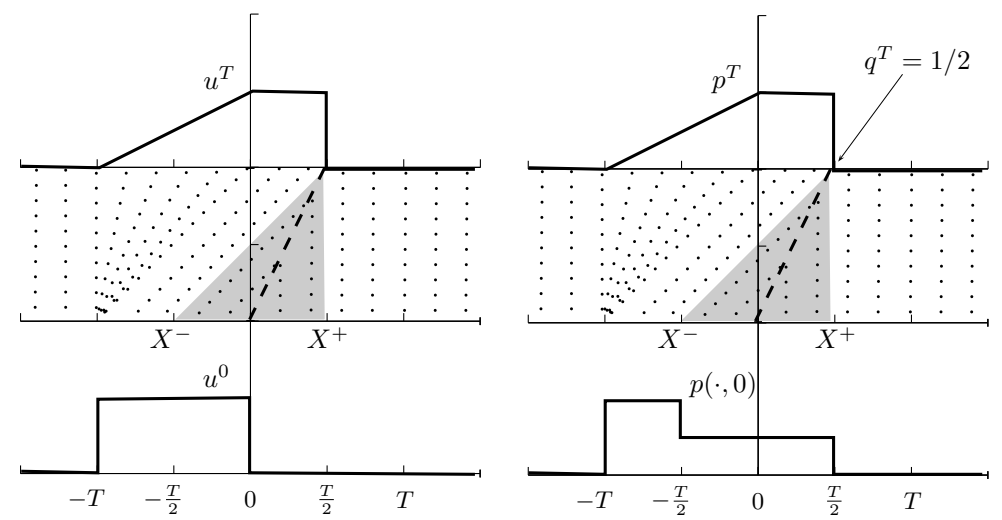

FiguRE 4. Schematic representation of the increase of the number of shocks in the Burgers' equation (1.5), when using the adjoint system. Here $u^{0} \in \mathcal{U}_{a d}, u^{d} \equiv 0$ and $q^{T}=-\left[u^{T}\right]_{\Sigma^{T}}$.

This is an important drawback in developing a descent algorithm for $J$. Indeed, according to the Definition 4.3 , if $\left(\delta u^{0}, \delta \varphi^{0}\right)$ is a descent direction belonging to $T_{u^{0}}$, the new datum $u^{0, \text { new }}$ should be obtained from $u^{0}$ following a path associated to this descent direction (see (4.12)).

Note that, if we take (4.45) as descent direction $\left(\delta u^{0}, \delta \varphi^{0}\right)$, which is not a generalized tangent vector as explained above, the new datum $u^{0, n e w}$ will have three discontinuities; the one coming from the displacement of the discontinuity of $u^{0}$ at 
$\Sigma^{0}$ and two more produced by the discontinuities of $p(x, 0)$. Thus, in an iterative process, the descent algorithm will create more and more discontinuities increasing artificially the complexity of solutions. This motivates the alternating descent method we propose here that, based on this notion of generalized gradient, develops a descent algorithm that keeps the complexity of solutions bounded. This will be done in the following Section.

We finish this section with the proof of Proposition 4.8.

Proof. (of Proposition 4.8) A straightforward computation shows that $J$ is Gâteaux differentiable in the generalized sense of Definition 4.7 and using Reynolds' transport theorem we obtain that, the generalized Gâteaux derivative of $J$ in the direction of the generalized tangent vector $\left(\delta u^{0}, \delta \varphi^{0}\right)$ is given by

$$
\begin{aligned}
\delta J\left(u^{0}\right)\left[\delta u^{0}, \delta \varphi^{0}\right]=\int_{\mathbb{R}^{2} \backslash \Sigma^{T}} & \left(u(x, T)-u^{d}(x)\right) \delta u(x, T) d x \\
& -\int_{\Sigma^{T}}\left[\frac{\left(u(\cdot, T)-u^{d}(\cdot)\right)^{2}}{2}\right]_{\Sigma^{T}}(x) \delta \varphi(x, T) d \Sigma^{T}(x),
\end{aligned}
$$

where the pair $(\delta u, \delta \varphi)$ solves the linearized problem (4.16)-(4.19) with initial data $\left(\delta u^{0}, \delta \varphi^{0}\right)$.

Let us now introduce the adjoint system (4.39)-(4.44). Multiplying the equations of $\delta u$ by $p$, and integrating we get

$$
\begin{aligned}
0= & \int_{\mathbb{R}^{2} \times(0, T)}\left(\partial_{t} \delta u+\operatorname{div}_{x}\left(f^{\prime}(u) \delta u\right)\right) p d x d t \\
=- & \int_{\mathbb{R}^{2} \times(0, T)} \delta u\left(\partial_{t} p+f^{\prime}(u) \nabla_{x} p\right) d x d t+\int_{\mathbb{R}^{2} \backslash \Sigma^{T}} \delta u(x, T) p(x, T) d x \\
& \quad-\int_{\mathbb{R}^{2} \backslash \Sigma^{0}} \delta u^{0}(x) p(x, 0) d x-\int_{\Sigma}\left([\delta u p]_{\Sigma^{t}} n_{\Sigma}^{t}+\left[f^{\prime}(u) \delta u p\right]_{\Sigma^{t}} \cdot n_{\Sigma}^{x}\right) d \Sigma .
\end{aligned}
$$

The first term in the right hand side vanishes since $p$ satisfies the adjoint equation (4.39).

Therefore, replacing (4.43), we obtain

$$
\begin{aligned}
& \text { (4.48) } \delta J\left(u^{0}\right)\left[\delta u^{0}, \delta \varphi^{0}\right]=\int_{\mathbb{R}^{2} \backslash \Sigma^{0}} \delta u^{0}(x) p(x, 0) d x \\
& +\int_{\Sigma}\left([\delta u p]_{\Sigma^{t}} n_{\Sigma}^{t}+\left[f^{\prime}(u) \delta u p\right]_{\Sigma^{t}} \cdot n_{\Sigma}^{x}\right) d \Sigma-\int_{\Sigma^{T}}\left[\frac{\left(u(\cdot, T)-u^{d}(\cdot)\right)^{2}}{2}\right]_{\Sigma^{T}}^{(x)} \delta \varphi(x, T) d \Sigma^{T}(x) .
\end{aligned}
$$

The last two terms in the right hand side of (4.48) will determine the conditions that $p$ must satisfy on the shock.

Observe that for any functions $f, g$ we have

$$
[f g]_{\Sigma^{t}}=\bar{f}[g]_{\Sigma^{t}}+\bar{g}[f]_{\Sigma^{t}}
$$

where $\bar{g}$ represents the average of $g$ to both sides of the shock $\Sigma^{t}$, i.e.

$$
\bar{g}(x, t)=\frac{1}{2} \lim _{\varepsilon \rightarrow 0}(g((x, t)+\varepsilon \hat{\nu})+g((x, t)-\varepsilon \hat{\nu})), \quad \forall(x, t) \in \Sigma .
$$


Thus we have

$$
\begin{aligned}
\int_{\Sigma}\left([\delta u p]_{\Sigma^{t}} n_{\Sigma}^{t}+\left[f^{\prime}(u) \delta u p\right]_{\Sigma^{t}} \cdot n_{\Sigma}^{x}\right) d \Sigma= & \int_{\Sigma}[p]_{\Sigma^{t}}\left(\overline{\delta u} n_{\Sigma}^{t}+\overline{f^{\prime}(u) \delta u} \cdot n_{\Sigma}^{x}\right) d \Sigma \\
& +\int_{\Sigma} \bar{p}\left([\delta u]_{\Sigma^{t}} n_{\Sigma}^{t}+\left[f^{\prime}(u) \delta u\right]_{\Sigma^{t}} \cdot n_{\Sigma}^{x}\right) d \Sigma .
\end{aligned}
$$

Using (4.17) and (4.40), we have

$$
\begin{aligned}
& \int_{\Sigma}\left([\delta u p]_{\Sigma^{t}} n_{\Sigma}^{t}+\left[f^{\prime}(u) \delta u p\right]_{\Sigma^{t}} n_{\Sigma}^{x}\right) d \Sigma= \\
& \int_{\Sigma} \bar{p} \operatorname{div}_{\Sigma}\left(\delta \varphi\left\|n_{\Sigma}^{x}\right\|\left([f(u)]_{\Sigma^{t}},[u]_{\Sigma^{t}}\right)\right) d \Sigma .
\end{aligned}
$$

On the other hand, we have the following identity

$$
\operatorname{div}_{\Sigma}(g G)=g \operatorname{div}_{\Sigma}(G)+\nabla_{\Sigma} g \cdot G,
$$

and from (4.30) and (4.37) we have the property

$$
\int_{\Sigma} \operatorname{div}_{\Sigma}\left(\left\|n_{\Sigma}^{x}\right\| G\right) d \Sigma=\int_{\Sigma^{T}} G \cdot \hat{k} d \Sigma^{T}-\int_{\Sigma^{0}} G \cdot \hat{k} d \Sigma^{0},
$$

for any $G \in\left(W^{1,1}(\Theta)\right)^{3}$, with $\Theta \subset \mathbb{R}^{2} \times \mathbb{R}$ an open set such that $\Sigma \subset \Theta$.

Thus, using (4.50) and (4.51) in (4.49) we obtain

$$
\begin{aligned}
\int_{\Sigma}\left([\delta u p]_{\Sigma^{t}} n_{\Sigma}^{t}+\left[f^{\prime}(u) \delta u p\right]_{\Sigma^{t}} n_{\Sigma}^{x}\right) d \Sigma= & \int_{\Sigma} \operatorname{div}_{\Sigma}\left(\bar{p} \delta \varphi\left\|n_{\Sigma}^{x}\right\|\left([f(u)]_{\Sigma^{t}},[u]_{\Sigma^{t}}\right)\right) d \Sigma \\
& -\int_{\Sigma} \delta \varphi\left\|n_{\Sigma}^{x}\right\|\left([f(u)]_{\Sigma^{t}},[u]_{\Sigma^{t}}\right) \cdot \nabla_{\Sigma} \bar{p} d \Sigma \\
= & \int_{\Sigma^{T}} \bar{p}(x, T) \delta \varphi(x, T)[u]_{\Sigma^{T}} d \Sigma^{T} \\
& -\int_{\Sigma^{0}} \bar{p}(x, 0) \delta \varphi(x, 0)[u]_{\Sigma^{0}} d \Sigma^{0} \\
& -\int_{\Sigma} \delta \varphi\left\|n_{\Sigma}^{x}\right\|\left([f(u)]_{\Sigma^{t}},[u]_{\Sigma^{t}}\right) \cdot \nabla_{\Sigma} \bar{p} d \Sigma .
\end{aligned}
$$

Therefore, replacing (4.41),(4.42),(4.44) and (4.52) in (4.48), we obtain

$$
\delta J\left(u^{0}\right)\left[\delta u^{0}, \delta \varphi^{0}\right]=\int_{\mathbb{R}^{2} \backslash \Sigma^{0}} \delta u^{0}(x) p(x, 0) d x-\int_{\Sigma^{0}} q(x, 0) \delta \varphi^{0}(x)[u]_{\Sigma^{0}} d \Sigma^{0} .
$$

This concludes the proof.

\section{The alternating Descent method}

As explained above, one of the main drawbacks of the continuous approach when dealing with discontinuities, is the increase in the complexity of solutions one gets along the iterative process, due to the use of optimal descent directions (see Figure 5 , for a scheme of the solution of the adjoint system, in this scheme we represent the behavior of the adjoint system in a toy $1 \mathrm{D}$ case, then in the new step we increase the number of discontinuities in $u^{0, \varepsilon}$ see Figure 6). A possible remedy is to use the generalized tangent vectors as descent directions for $J$. 

$(5.1)$

First let us introduce some notation. We consider two curves $X^{-}=\left\{x-T f^{\prime}\left(u^{-}(x, T)\right) \mid \forall x \in \Sigma^{T}\right\}, \quad X^{+}=\left\{x-T f^{\prime}\left(u^{+}(x, T)\right) \mid \forall x \in \Sigma^{T}\right\}$, and the set $\left[X^{-}, \Sigma^{0}\right]$ constituted by the points in $\mathbb{R}^{2}$ between the curves $X^{-}$and $\Sigma^{0}$. We can also define similarly the set $\left[\Sigma^{0}, X^{+}\right]$.

The set $\left[X^{-}, X^{+}\right]$represents the basis of the black triangle in Figures 3 and 4 , where shocks collapse at time $t=T$.
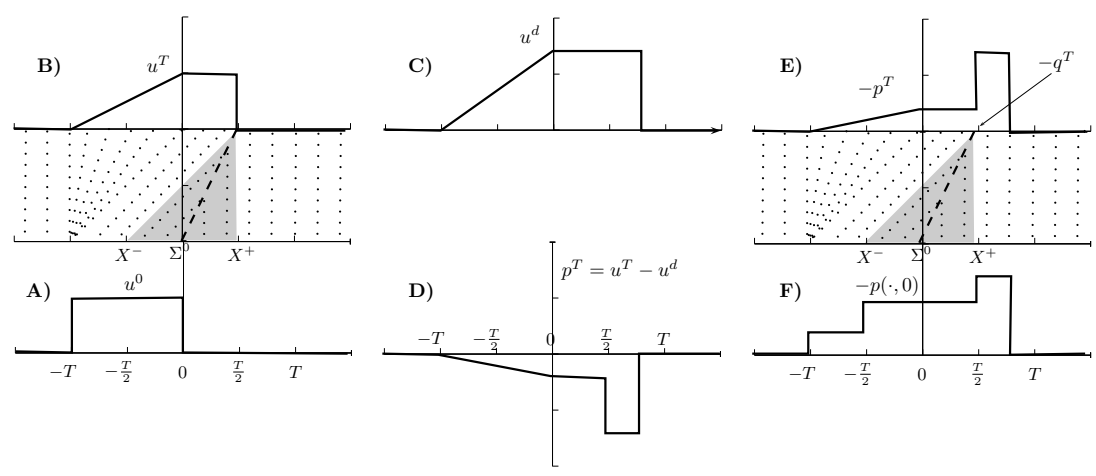

FIGURE 5. Scheme of the solution of the adjoint system in a toy case. A) The initial datum $u^{0}$. B) The solution of the Burgers' equation at time $T$. C) The target function $u^{d}$. D) The value at $t=T$ of the adjoint state: $p^{T}(x)=u(x, T)-u^{d}(x)$. E) The initial conditions $p^{T}$ and $q^{T}$. F) The solution of the adjoint system at time $t=0$.
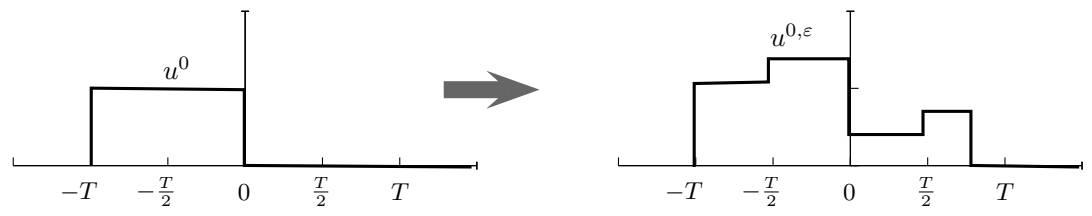

FigurE 6. Scheme of the next step in the descent process.

5.1. The algorithm in 2D. Following the previous ideas, we introduce descent directions that do not increase the complexity of the solution in each iteration of the optimization process.

We now introduce the three classes of perturbations we shall use in our descent algorithm.

First class of perturbations: With this set of perturbations we want to change the profile of $u^{0}$ only at one side of the shock. We set the first 
perturbation $d_{1}=\left(\delta u^{0}, \delta \varphi^{0}\right)$, given by:

$$
\delta \varphi^{0} \equiv 0, \quad \delta u^{0}(x)=\left\{\begin{array}{cc}
-p(x, 0), & x \in Q_{-}^{0} \backslash\left[X^{-}, \Sigma^{0}\right] \\
r(x), & x \in\left[X^{-}, \Sigma^{0}\right] \\
0, & \text { otherwise }
\end{array}\right.
$$

where $p$ is the solution of the adjoint system (4.39)-(4.43) and $r(x)$ is any extension of $-p(x, 0)$ to the set $\left[X^{-}, \Sigma^{0}\right]$ such that $\delta u^{0}$ is only discontinuous on $\Sigma^{0}$.

Second class of perturbations: In this case, we only want to move the shock without changing the profile of the function. We set the second perturbation $d_{2}=\left(\delta u^{0}, \delta \varphi^{0}\right)$, given by:

$$
\delta u^{0} \equiv 0, \quad \delta \varphi^{0}(x)=\frac{q(x, 0)}{[u]_{\Sigma^{0}}},
$$

where $q$ is the solution of the adjoint system (4.42),(4.44).

Third class of perturbations: With this set of perturbations we want to change the profile of $u^{0}$ only at one side of the shock, without modifying the position of the shock at time $t=0$. The third perturbation $d_{3}=\left(\delta u^{0}, \delta \varphi^{0}\right)$ is chosen to be:

$$
\delta \varphi^{0} \equiv 0, \quad \delta u^{0}(x)=\left\{\begin{array}{cc}
-p(x, 0) & x \in Q_{+}^{0} \backslash\left[\Sigma^{0}, X^{+}\right] \\
r(x) & x \in\left[\Sigma^{0}, X^{+}\right] \\
0 & \text { otherwise }
\end{array}\right.
$$

where $p$ is the solution of the adjoint system (4.39)-(4.43) and $r(x)$ is any extension of $-p(x, 0)$ to the set $\left[\Sigma^{0}, X^{+}\right]$such that $\delta u^{0}$ is only discontinuous on $\Sigma^{0}$.

In this way the first and the third perturbations will conserve the shock structure and only change the profile of the solution to both sides. Therefore $d_{1}$ and $d_{3}$ given by $(5.2),(5.4)$ satisfy

$$
\begin{aligned}
& \delta J\left(u^{0}\right)\left[d_{1}\right]=-\int_{Q_{-}^{0} \backslash\left[X^{-}, \Sigma^{0}\right]}|p(x, 0)|^{2} d x+\int_{\left[X^{-}, \Sigma^{0}\right]} r(x) p(x, 0) d x, \\
& \delta J\left(u^{0}\right)\left[d_{3}\right]=-\int_{Q_{+}^{0} \backslash\left[\Sigma^{0}, X^{+}\right]}|p(x, 0)|^{2} d x+\int_{\left[\Sigma^{0}, X^{+}\right]} r(x) p(x, 0) d x .
\end{aligned}
$$

Then, in general, these classes of perturbations do not necessarily produce the decrease of the functional $J$, but they preserve the structure of the function $u^{0}$ and, simultaneously, give the possibility of moving from a local minimizer to a global one, exploring other profiles outside of the shock. However, the second perturbation $d_{2}$ given by (5.3) satisfies

$$
\delta J\left(u^{0}\right)\left[d_{2}\right]=-\int_{\Sigma 0}|q(x, 0)|^{2} d \Sigma^{0} \leq 0 .
$$

Thus $d_{2}$ produces the decrease of the functional $J$ and preserves the structure of the function $u^{0}$.

Remark 5.1. When considering the first and third perturbation, we need to choose $r$. This corresponds to the inpainting (image interpolation) problem in image restoration. Following the approaches by Rudin, Osher and Fatemi [31] and the objectedge model by Mumford and Shah [30], we may take $r$ as the solution of a general 


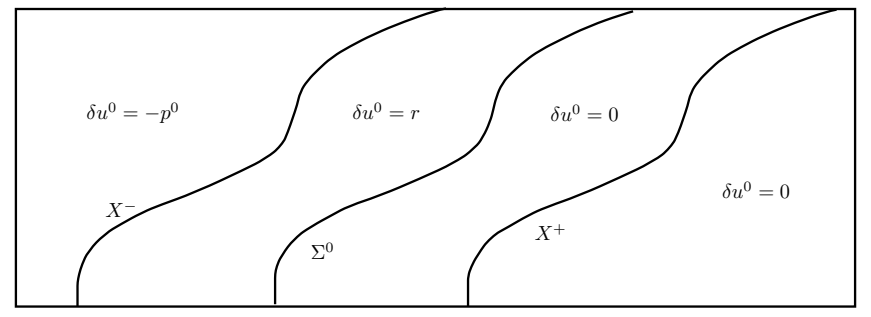

Figure 7. Scheme of the curves $\Sigma^{0}, X^{-}, X^{+}$, and the extension of $\delta u^{0}$ in the first perturbation.

variational inpainting model, minimizing a variational problem of the form:

$$
\min _{\psi \in W^{1,2}\left(\mathbb{R}^{2}\right)} E(\psi)=\frac{\alpha}{2} \int_{\Omega \backslash\left[X^{-}, X^{+}\right]}\left|\psi+p^{0}\right|^{2} d x+\frac{\beta}{2} \int_{\Omega}|\nabla \psi|^{2} d x,
$$

$\Omega=Q_{-}^{0}$ being fixed for the first perturbation and $\Omega=Q_{+}^{0}$ for the third perturbation. Here $\alpha$ and $\beta$ are positive constants. In (5.8) the first term is commonly called the data fidelity term, that represents the data assimilation of the model and the last term gives the regularity of the solution, producing a good interpolation. Whether there are other, better suited, methods to extend $\delta u^{0}$ near the shock is an interesting open problem.

We observe that the choice of $r$ is not relevant at first order outside the shock. Indeed, $u^{0}$ depends on $r$ only in $\left[X^{-}, X^{+}\right]$and in the linear system (4.16), (4.17), (4.18), (4.19), this region at time $T$ collapses on the shock curve $\Sigma^{T}$. Therefore, the choice of $r$ only affects the motion of the curve $\Sigma^{T}$ at time $T$. Then when we fixe $r$, we loose the optimal descent direction for the functional $J$ but we do not change essentially the profile of the solution $u(\cdot, T)$ at both sides of the shock. We mainly affect the position of the shock $\Sigma^{T}$, because this region $\left[X^{-}, X^{+}\right]$collapses at time $T$.

5.2. Descent strategy. Here we propose a new method built on the results in Proposition 4.8 and the discussion thereafter, which is an extension to $2 \mathrm{D}$ of the method introduced in [12]. We shall refer to this new method as the alternating descent method in $2 \mathrm{D}$.

For a given initialization of $u^{0}$, in each step of the descent iteration process, we proceed in the following three sub-steps:

(1) First direction: We change the profile in $Q_{-}^{0}$.

- Compute (5.2) and find the optimal step size $\varepsilon$ such that $p(x, 0)$, the solution of the adjoint system (4.39)-(4.43) and its $L^{2}$-norm diminish in $Q_{-}^{0} \backslash\left[X^{-}, \Sigma^{0}\right]$. In this way we obtain the best profile in $Q_{-}^{0}$ for $u^{0}$.

- Compute (5.3) and find the optimal step size $\varepsilon$ for which this datum must be modified as in (5.3). In this way we obtain the best location of the discontinuity for this $u^{0}$.

(2) Second direction: We only move the shock. Compute (5.3) and find the optimal step size $\varepsilon$ for which this datum must be modified in perturbations given by (5.3). 
(3) Third direction: We change the profile in $Q_{+}^{0}$.

- Compute (5.4) and find the optimal step size $\varepsilon$ such that $p(x, 0)$ the solution of the adjoint system (4.39)-(4.43) its $L^{2}$-norm is diminishing in $Q_{+}^{0} \backslash\left[\Sigma^{0}, X^{+}\right]$. In this way we obtain the best profile in $Q_{+}^{0}$ for $u^{0}$.

- Compute (5.3) and find the optimal step size $\varepsilon$ for which this datum must be modified in the perturbation given by (5.3).

In the above procedure, finding the step size $\varepsilon$ involves a one-dimensional optimization problem that we can solve with a classical method (bisection, Armijo's rule, etc.). In the first and third directions we choose the step size $\varepsilon$ such that the $L^{2}$-norm of $p(\cdot, 0)$ decreases, trying to move to a critical point of the functional $J$.

If we consider $u^{0}$ given by (5.2) or (5.4), we can solve (4.16),(4.18) and compute the right side of the equation (4.17). Therefore, using (4.37), we have that, under the hypothesis (4.2), the coefficients in the equation (4.17) are Lipschitz continuous. Then we observe that there exists $\delta \varphi^{0}$ such that,

$$
\begin{aligned}
\operatorname{div}_{\Sigma}\left(\delta \varphi\left\|n_{\Sigma}^{x}\right\|\left([f(u)]_{\Sigma^{t}},[u]_{\Sigma^{t}}\right)\right) & =\left(\left[f^{\prime}(u) \delta u\right]_{\Sigma^{t}},[\delta u]_{\Sigma^{t}}\right) \cdot n_{\Sigma}, & & \text { on } \Sigma \\
\delta \varphi(x, 0) & =\delta \varphi^{0}(x), & & x \in \Sigma^{0},
\end{aligned}
$$

satisfies the terminal condition:

$$
\delta \varphi(x, T)=0, \quad x \in \Sigma^{T} .
$$

Therefore, the first and third directions produce the decrease of the functional $J$ at first order.

Thus, one has to iterate this procedure to assure a simultaneous better placement of the shock and a better fitting of the value of the solution away from it.

The main difference of our 2D algorithm with the 1D one proposed in [12] relies on the definition of the first and third classes of directions. In [12] $\delta \varphi^{0}$ does not necessarily vanish, but is rather taken for the shock at time $T$ not to move. The analogue in $2 \mathrm{D}$ would be to find $\delta \varphi^{0}$ in such a way that the solution of (5.9) satisfies $\delta \varphi^{T} \equiv 0$ (5.10). This can be viewed as a null control problem, the control being $\delta \varphi^{0}$. And for this choice of $\delta \varphi^{0}, \delta J\left(u^{0}\right)\left[\delta u^{0}, \delta \varphi^{0}\right] \leq 0$. But the computation of the solution of this control problem (5.9) is expensive, since it requires to obtain the coefficients of (5.9), i.e. $u$ and $\delta u$ at both sides of $\Sigma$, and then to develop a numerical solver for the control of (5.9). For that reason we only consider the perturbations introduced in (5.2),(5.4). These directions conserve the structure of the shock and change the profile of the solution. See Figures 8, 9, 10 and 11 for a numerical example where the first perturbation is computed.

In the next section we explain how to implement a descent algorithm following these ideas.

\section{Numerical approximation of the Descent DiReCtion}

We have computed the gradient of the continuous functional $J$ in several cases ( $u$ smooth or having shock discontinuities) but, in practice, one has to look for descent directions for a discrete version of the functional $J$. In this section we discuss two possibilities for searching them based either on the discrete or the continuous approaches.

The discrete approach consists mainly in applying a descent algorithm to the discrete version $J_{\Delta}$ of the functional $J$. The alternating descent method, by the contrary, is a continuous method based on the analysis of the previous section in which the two main classes of directions are identified. 


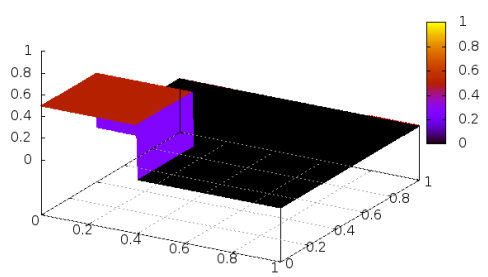

FIGURE 8. Initial condition $u^{0}$

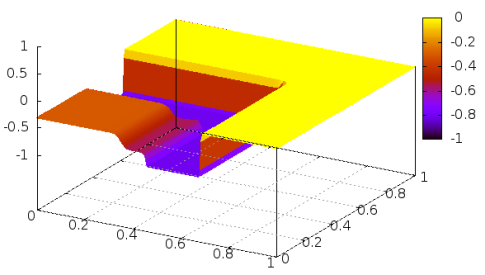

Figure 10. Solution of the adjoint system $p^{0}$

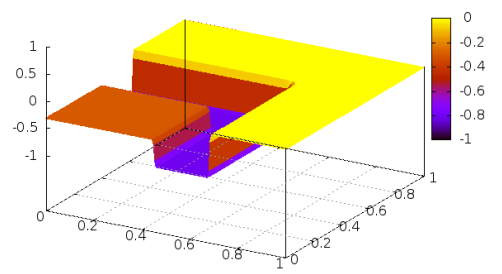

Figure 9. Datum of the adjoint system at time $t=T, p^{T}$

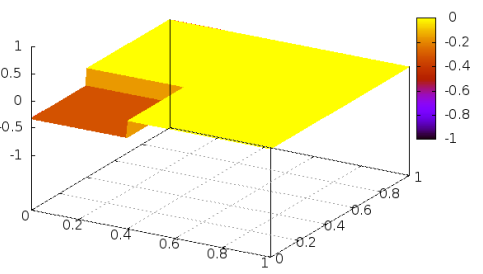

Figure 11. First direction $\delta u^{0}$

Let us first discuss the discrete approach.

6.1. The discrete approach. Let us consider the approximation of the functional $J$ by $J_{\Delta}$ defined (1.1) and (3.1) respectively. Consider a 3 -point conservative numerical approximation scheme for (1.2), using a dimensional splitting in both variables, i.e. we use (3.17) and (3.15) with in each component $H_{\Delta}^{x}$ and $H_{\Delta}^{y}$. We shall use a 1D Engquist-Osher scheme, with the following numerical flux:

$$
\begin{aligned}
g_{1}(a, b) & =\frac{1}{2}\left(f_{1}(a)+f_{1}(b)-\int_{b}^{a}\left|f_{1}^{\prime}(z)\right| d z\right) \\
g_{2}(a, b) & =\frac{1}{2}\left(f_{2}(a)+f_{2}(b)-\int_{b}^{a}\left|f_{2}^{\prime}(z)\right| d z\right) .
\end{aligned}
$$

More explicitly, replacing (3.15) in (3.17), we obtain

$$
\begin{aligned}
& u_{i, j}^{n+\frac{1}{2}}=u_{i, j}^{n}-\frac{\Delta t}{\Delta x}\left(g_{1}\left(u_{i+1, j}^{n}, u_{i, j}^{n}\right)-g_{1}\left(u_{i, j}^{n}, u_{i-1, j}^{n}\right)\right), \\
& i \in \mathbb{Z}, n=0, \ldots, N \\
& u_{i, j}^{n+1}=u_{i, j}^{n+\frac{1}{2}}-\frac{\Delta t}{\Delta y}\left(g_{2}\left(u_{i, j+1}^{n+\frac{1}{2}}, u_{i, j}^{n+\frac{1}{2}}\right)-g_{2}\left(u_{i, j}^{n+\frac{1}{2}}, u_{i, j-1}^{n+\frac{1}{2}}\right)\right), \\
& j \in \mathbb{Z}, n=0, \ldots, N,
\end{aligned}
$$


where $g_{1}, g_{2}$ are the numerical fluxes defined in (6.1).

The gradient of the discrete functional $J_{\Delta}$ requires computing one derivative of $J_{\Delta}$ with respect to each node of the mesh. This can be done in a cheaper way using the adjoint state. We illustrate it for the Engquist-Osher numerical scheme. However, as the discrete functionals $J_{\Delta}$ are not necessarily convex the gradient methods could possibly provide sequences that do not converge to a global minimizer of $J_{\Delta}$. But this drawback and difficulty appears in most applications of descent methods in optimal design and control problems. As we will see, in the present context, the approximations obtained by gradient methods are satisfactory, although convergence is slow due to unnecessary oscillations that the descent method introduces. The gradient of $J_{\Delta}$, rigorously speaking, requires the linearization of the numerical scheme (6.2),(6.3) used to approximate the equation (1.2). Then the linearization corresponds to

$$
\begin{aligned}
\delta u_{i, j}^{n+\frac{1}{2}}= & \delta u_{i, j}^{n}-\frac{\Delta t}{\Delta x}\left(\partial_{a} g_{1}\left(u_{i+1, j}^{n}, u_{i, j}^{n}\right) \delta u_{i+1, j}^{n}-\partial_{b} g_{1}\left(u_{i, j}^{n}, u_{i-1, j}^{n}\right) \delta u_{i-1, j}^{n}\right) \\
& -\frac{\Delta t}{\Delta x}\left(\partial_{b} g_{1}\left(u_{i+1, j}^{n}, u_{i, j}^{n}\right)-\partial_{a} g_{1}\left(u_{i, j}^{n}, u_{i-1, j}^{n}\right)\right) \delta u_{i, j}^{n}, \\
(6.4) \quad & i, j \in \mathbb{Z}, \quad n=0, \ldots, N, \\
\delta u_{i, j}^{n+1}= & \delta u_{i, j}^{n+\frac{1}{2}}-\frac{\Delta t}{\Delta y}\left(\partial_{a} g_{2}\left(u_{i, j+1}^{n+\frac{1}{2}}, u_{i, j}^{n+\frac{1}{2}}\right) \delta u_{i, j+1}^{n+\frac{1}{2}}-\partial_{b} g_{2}\left(u_{i, j}^{n+\frac{1}{2}}, u_{i, j-1}^{n+\frac{1}{2}}\right) \delta u_{i, j-1}^{n+\frac{1}{2}}\right) \\
& -\frac{\Delta t}{\Delta y}\left(\partial_{b} g_{2}\left(u_{i, j+1}^{n+\frac{1}{2}}, u_{i, j}^{n+\frac{1}{2}}\right)-\partial_{a} g_{2}\left(u_{i, j}^{n+\frac{1}{2}}, u_{i, j-1}^{n+\frac{1}{2}}\right)\right) \delta u_{i, j}^{n+\frac{1}{2}}, \\
(6.5) \quad & i, j \in \mathbb{Z}, \quad n=0, \ldots, N .
\end{aligned}
$$

In view of this, the discrete adjoint system of (6.4),(6.5) can also be written for the differentiable flux functions (6.1):

$$
\begin{aligned}
p_{i, j}^{N+1}= & p_{i, j}^{T}, \quad i, j \in \mathbb{Z}, \\
p_{i, j}^{n+\frac{1}{2}}= & p_{i, j}^{n+1}+\frac{\Delta t}{\Delta y} \partial_{b} g_{2}\left(u_{i, j+1}^{n+\frac{1}{2}}, u_{i, j}^{n+\frac{1}{2}}\right)\left(p_{i, j+1}^{n+1}-p_{i, j}^{n+1}\right) \\
& +\frac{\Delta t}{\Delta y} \partial_{a} g_{2}\left(u_{i, j}^{n+\frac{1}{2}}, u_{i, j-1}^{n+\frac{1}{2}}\right)\left(p_{i, j 1}^{n+1}-p_{i, j-1}^{n+1}\right), \\
& i, j \in \mathbb{Z}, \quad n=0, \ldots, N, \\
p_{i, j}^{n}= & p_{i, j}^{n+\frac{1}{2}}+\frac{\Delta t}{\Delta x} \partial_{b} g_{1}\left(u_{i+1, j}^{n}, u_{i, j}^{n}\right)\left(p_{i+1, j}^{n+\frac{1}{2}}-p_{i, j}^{n+\frac{1}{2}}\right) \\
& +\frac{\Delta t}{\Delta x} \partial_{a} g_{1}\left(u_{i, j}^{n}, u_{i-1, j}^{n}\right)\left(p_{i, j}^{n+\frac{1}{2}}-p_{i-1, j}^{n+\frac{1}{2}}\right), \\
& i, j \in \mathbb{Z}, \quad n=0, \ldots, N .
\end{aligned}
$$

In fact, when multiplying the equations in (6.5) by $p_{i, j}^{n+1}$ and adding in $i, j \in \mathbb{Z}$ and $n=0, \ldots, N$, the following identity is easily obtained,

$$
\Delta x \Delta y \sum_{i, j \in \mathbb{Z}} p_{i, j}^{T} \delta u_{i, j}^{N+1}=\Delta x \Delta y \sum_{i, j \in \mathbb{Z}} p_{i, j}^{0} \delta u_{i, j}^{0}
$$

This is the discrete version of formula (4.4) which allows us to simplify the derivative of the discrete cost functional. 
Thus, for any variation $\delta u_{\Delta}^{0}$, the Gâteaux derivative of the cost functional defined in (3.1) is given by

$$
\delta J_{\Delta}=\Delta x \Delta y \sum_{i, j \in \mathbb{Z}}\left(u_{i, j}^{N+1}-u_{i, j}^{d}\right) \delta u_{i, j}^{N+1},
$$

where $\delta u_{i, j}^{n}$ solves the linearized system $(6.4),(6.5)$. If we consider $p_{i, j}^{n}$ the solution of $(6.6),(6.8),(6.10)$ with final datum

$$
p_{i, j}^{T}=u_{i, j}^{N+1}-u_{i, j}^{d}, \quad \forall i, j \in \mathbb{Z},
$$

then $\delta J_{\Delta}$ in $(6.12)$ can be written as,

$$
\delta J_{\Delta}=\Delta x \Delta y \sum_{i, j \in \mathbb{Z}} p_{i, j}^{0} \delta u_{i, j}^{0}
$$

and this allows to obtain easily the steepest descent direction for $J_{\Delta}$ by considering

$$
\delta u_{\Delta}^{0}=-p_{\Delta}^{0}
$$

Remark 6.1. We do not address here the problem of the convergence of this adjoint scheme towards the solution of the continuous adjoint system. Of course, this is an easy matter when $u$ is smooth but it is far from being trivial when $u$ has shock discontinuities. Whether or not this discrete adjoint system, as $\Delta \rightarrow 0$, allows reconstructing the complete adjoint system, with the inner Dirichlet condition along the shock (4.39),(4.40),(4.41),(4.42),(4.43) and (4.44), constitutes an interesting problem for future research. We refer to [25] and [34] for preliminary work on this direction in one-dimension.

6.2. The alternating descent method in 2D. Now we explain how we implement the method proposed in Subsection 5.2. The main idea is to approximate a minimizer of $J$ alternating with three directions: First we perturb the initial datum $u^{0}$ at side $Q_{-}^{0}$ and find the motion of the curve which produces the decrease of $J$. Second we move the shock curve without altering the profile of $u^{0}$ at both sides of $\Sigma$. Finally the third direction perturbs the profile in $Q_{+}^{0}$ and we find the motion of the curve which produces the decrease of $J$.

More precisely, for a given initialization $u^{0}$ and target function $u^{d}$, we implement the following Algorithm 1, iterating it until we reach the stopping criterion of the minimization process.

The main advantage of this method is that for an initial datum $u^{0}$ with a single discontinuity, the descent directions are generalized tangent vectors, i.e. they introduce Lipschitz continuous variations of $u^{0}$ at both sides of the discontinuity and a displacement of the shock position. In this way, the new datum obtained modifying the old one, in the direction of this generalized tangent vector, will have again a single discontinuity. We have presented here the method in the particular case in which both the target $u^{d}$ and the initial datum $u^{0}$ that initializes the process have one single shock discontinuity. But these ideas can be applied in a much more general context in which the number of shocks does not necessarily coincide. In particular, in one dimension this method is able both to generate shocks and to destroy them, if any of these facts contributes to the decrease of the functional. This method is in some sense close to those employed in shape design in elasticity in which topological derivatives (that in the present setting would correspond to 


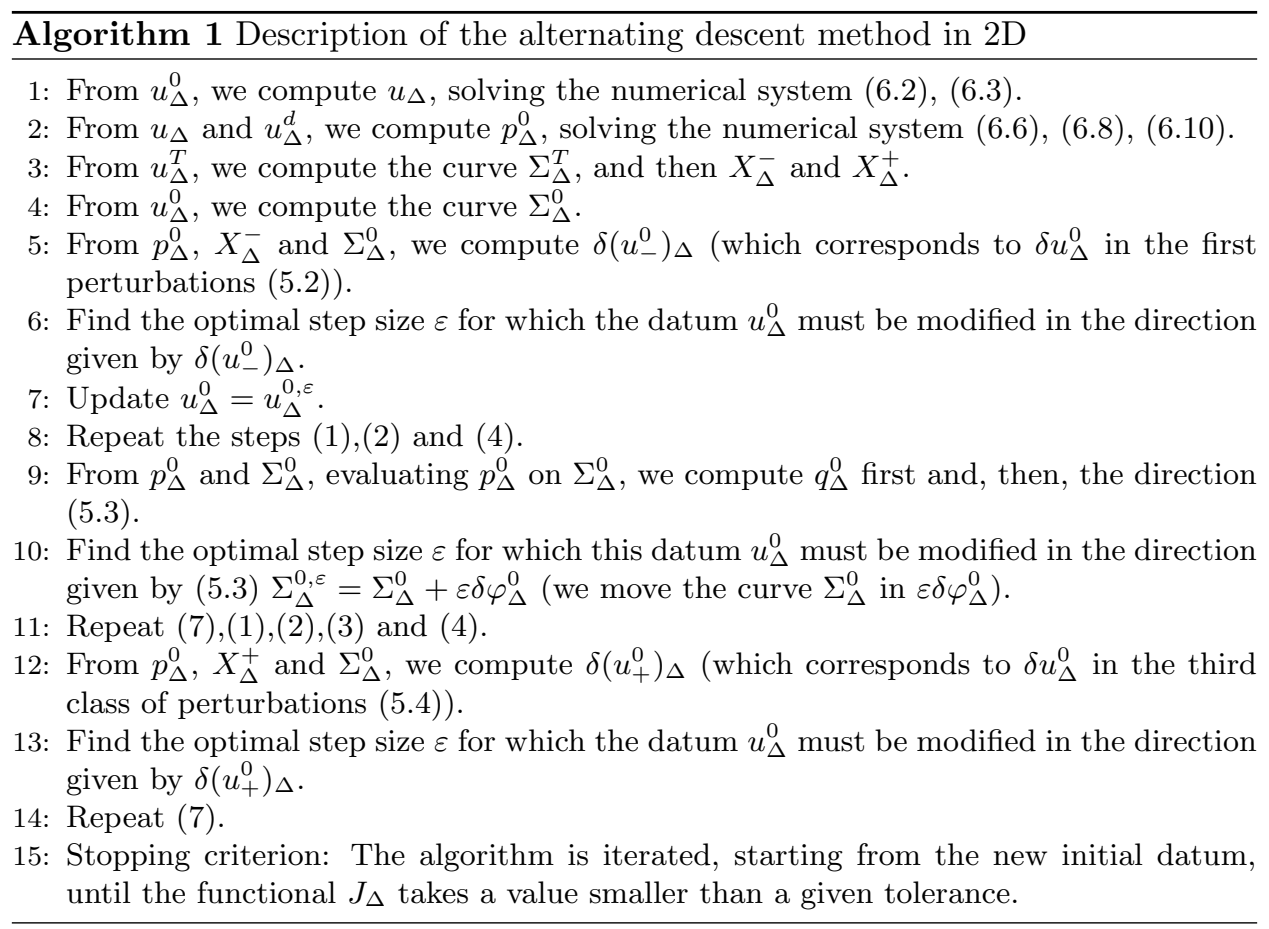

controlling the location of the shock) are combined with classical shape deformations (that would correspond to simply shaping the solution away from the shock in the present setting) [20].

\section{NumericAl implementation}

In this section we explain the main computational ingredients entering in the implementation of the alternating descent method in 2D, and its solutions from a numerical point of view.

The first difficulty arises when determining the boundary conditions on the numerical scheme (especially if the shock interacts with the boundary): The behaviour of boundary conditions is well understood for 1D scalar problems, but their treatment is less clear for systems and in 2D (Dubois and LeFloch, 1988 [17]). For that reason we employ a ghost cell strategy in our numerical schemes. Using the maximum principle and the finite velocity of propagation that entropy solutions satisfy we obtain an efficient numerical implementation. The ghost cell strategy consists in considering a larger computational domain, containing the support of the solution $\left\{u_{\Delta}^{n}\right\}_{n=0, \ldots, N}$ of $(6.2),(6.3)$ for all $n=0, \ldots, N+1$. Its size can be estimated from the initial condition $u_{\Delta}^{0}$, thanks to the finite velocity of propagation. The functional $J_{\Delta}$ is then localized in a smaller domain such that, the boundary effects in the larger one do not affect its value.

On the other hand, we did no develop a specific method to approximate the continuous adjoint system. We rather use the discrete adjoint as an approximation, although its validity is not analytically established.

Now, we comment the main difficulties encountered in the implementation of the Algorithm 1 and the way we overcome them: 
Edge detector: One of the key steps in the implementation of the alternating descent method is the identification of the shock location. This requires a numerical method to recover the curves $\Sigma^{T}$ and $\Sigma^{0}$. We describe a subroutine to find the discontinuities of a vector $u_{\Delta}=\left\{u_{i, j}\right\}_{i, j=1, \ldots, N}$ based on a shift condition. Roughly, we introduce a parameter $\alpha$ and we look for the indexes $i, j \in\{1, \ldots, N-2\}$, where

$D_{\Delta}\left(u_{\Delta}, i, j\right)>\alpha$, and $\left(D_{\Delta}\left(u_{\Delta}, i+1, j\right) \leq \alpha\right.$, or $\left.D_{\Delta}\left(u_{\Delta}, i, j+1\right) \leq \alpha\right)$,

where $D_{\Delta}\left(u_{\Delta}, i, j\right)=\left|u_{i+1, j}-u_{i, j}\right|+\left|u_{i, j+1}-u_{i, j}\right|$. The condition

$$
D_{\Delta}\left(u_{\Delta}, i, j\right)>\alpha,
$$

means that the discrete gradient is greater than $\alpha$ in the node $i, j$. In this way, we obtain a region of nodes fulfilling this condition. The other condition in (7.1) is to identify a curve and not a region. To simplify the presentation we consider the case in which only one discontinuity curve is relevant in the numerical experiment, that we identify on the discrete collection of the indexes by the above criterium. See Algorithm 2 for more details. For a more sophisticated algorithm see [11].

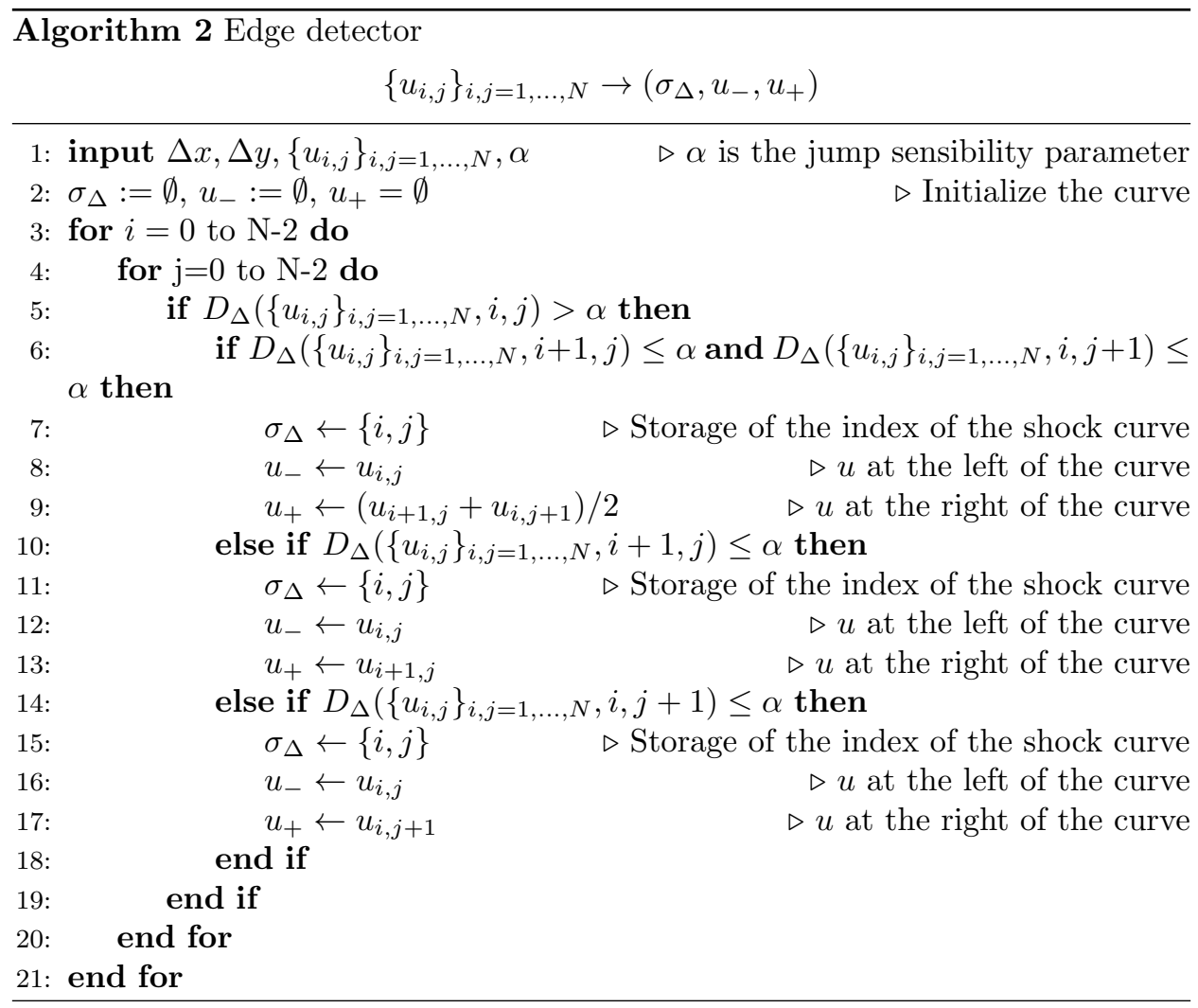

Segmentation problem: In lines 5 and 12 of Algorithm 1 it is necessary to restrict the function $p^{0}$ to the regions $\left[X^{-}, \Sigma^{0}\right]$ and $\left[\Sigma^{0}, X^{+}\right]$respectively. This requires an algorithm to identify whether a point is to the right or 
to left side of a given curve. This is a segmentation problem in image processing that we solve using the winding number algorithm. $w(a)$, the winding number of a closed curve (polygon in the discrete case) $C$ about a point $a$ in the plane, is defined as a contour integral in the complex plane:

$$
W(a)=\frac{1}{2 \pi i} \oint_{C} \frac{1}{z-a} d z
$$

that measures not only whether $C$ encloses $a$, but also how many times and in which orientation $C$ "winds around" $a$. In particular

$w(a)=\left\{\begin{array}{cl}0 & \text { if } a \text { is not inside } C \\ n>0 & \text { if } C \text { winds around } a n \text { times counterclockwise } \\ n<0 & \text { if } C \text { winds around } a-n \text { times clockwise. }\end{array}\right.$

Note that the winding number is not defined when the point $a$ is on the curve $C$. The winding number algorithm consists in computing, for a given point $a$, the winding number $w(a)$ with respect to the curve $C$ (oriented counterclockwise). If the winding number $w(a)$ is positive, the point lies inside the curve. There exists various versions of this algorithm employing different discretization of the above integral. For more details see [16], [26].

Inpainting problem: In the steps 5 and 12 in the Algorithm 1 we need to extend the function $p(\cdot, x)$ in the corresponding regions. As in Remark 5.1 we see there exists a rich literature on this problem. The common strategy is to consider the minimization of a discrete version of a variational problem (5.8) and to apply a descent algorithm, for example the steepest descent algorithm.

For our examples in the next section, $-p^{0}$ is a constant step function. And a strategy to extend $-p^{0}$ on $\left[X^{-}, \Sigma^{0}\right]$ and over $\left[\Sigma^{0}, X^{+}\right]$, such that, the extension only has a shock on $\Sigma^{0}$, consists in extending the constant value inside the corresponding regions.

Motion and deformation of the shock curve: To produce the motion of the curve, we use the Fast Marching Method introduced by J. A. Sethian see [32]. After moving the shock curve, we need to change the value of the profile $u^{0}$ at both sides of the new curve, following the normal lines, using a discrete version of (4.12).

\section{Numerical EXPERIMENTS}

In this section we present two numerical experiments illustrating the results obtained in an optimization model problem with each one of the numerical methods described in the previous section. We have chosen as computational domain the square $(0,1) \times(0,1)$.

8.1. Experiment 1. In this experiment we consider the conservation law

$$
\partial_{t} u+\left(u^{2} / 2\right)_{x}+\left(u^{4} / 4\right)_{x}=0, \text { in } \mathbb{R}^{2} \times(0, T),
$$

the time horizon $T=0.2$ and the target function $u^{d}$ given by

$$
u^{d}(x, y)=\left\{\begin{array}{cl}
0.7 & x \leq 0.87, \quad y \leq 0.76715 \\
0 & \text { otherwise }
\end{array}\right.
$$

To initialize the iterative descent method we choose an initial datum $u^{0}$ in such a way that the solution at time $t=T$ has a profile similar to $u^{d}$, i.e., it is a Lipschitz 
continuous function with a single discontinuity, located on a curve $\Sigma^{0} \subset \mathbb{R}^{2}$. For example

$$
u^{0}(x, y)=\left\{\begin{array}{cl}
0.4 & x \leq 0.2, \quad y \leq 0.4 \\
0 & \text { otherwise. }
\end{array}\right.
$$

We fix the computational domain $(0,1) \times(0,1)$, and the mesh size $\Delta x=\Delta y=$ $\Delta t=1 / 200$.

The CFL-condition for the numerical scheme $(6.2),(6.3)$ is

$$
\max \left\{\frac{\Delta t}{\Delta x} \sup _{x \in \mathbb{R}}\left|f_{1}^{\prime}\left(u^{0}(x)\right)\right|, \frac{\Delta t}{\Delta y} \sup _{x \in \mathbb{R}}\left|f_{2}^{\prime}\left(u^{0}(x)\right)\right|\right\} \leq 1,
$$

where $f(z)=\left(f_{1}(z), f_{2}(z)\right)$. Thus, the CFL-condition, in our experiment corresponds to

$$
\max \left\{\sup _{x \in \mathbb{R}}\left|u^{0}(x)\right|, \sup _{x \in \mathbb{R}}\left|u^{0}(x)\right|^{3}\right\} \leq 1 .
$$

Therefore $u^{0}$ given by (8.3), satisfies (8.5).

In this form, one minimizer is

$$
u_{*}^{0}(x, y)=\left\{\begin{array}{cl}
0.7 & x \leq 0.8, \quad y \leq 0.75 \\
0 & \text { otherwise. }
\end{array}\right.
$$

In Figure 12 we plot $\log (J)$ with respect to the number of iterations, for both, the alternating descent method proposed in this article and the purely discrete approach. We see that the method introduced in this work stabilizes in fewer iterations.

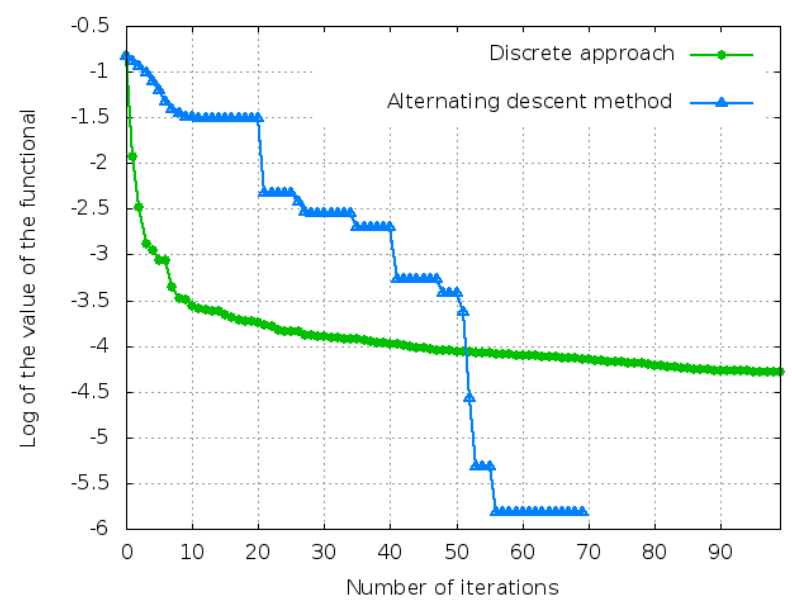

Figure 12. Experiment 1. Log of the value of the functional versus the number of iterations in the descent algorithm for the discrete approach scheme and the $2 \mathrm{D}$ alternating descent method.

In the Figures 16 and 17, we observe the minimizers obtained by the methods above, and the associated solutions in time T, Figures 18 and 19.

The function $u^{0}$ obtained by the alternating descent method (Figures 16) is a good approximation of (8.6). The solution given by the discrete approach (Figures 
17) presents high oscillations and this can produce problems with the CFL-condition (8.5). In Figures 17 and 19 we observe that the solution of the discrete approach preserves a memory of the initialization $u^{0}$ in (8.3), i.e. the solution of the discrete approach has oscillations around the shock location of the initial condition $u^{0}$ in $(8.3)$.

8.2. Experiment 2. In this experiment we consider the same conservation law (8.1) and the horizon time $T=0.2$. The target function $u^{d}$ corresponds to the solution of the numerical scheme $(6.2),(6.3)$ with the initial condition given by

$$
u_{*}^{0}(x, y)=\left\{\begin{array}{cl}
0.7 & (x, y) \notin \mathbb{R}_{+} \times \mathbb{R}_{+}, x \leq 0.7, y \leq 0.7 \\
0.7 & (x, y) \in \mathbb{R}_{+} \times \mathbb{R}_{+}, x^{2}+y^{2} \leq(0.7)^{2} \\
0 & \text { otherwise. }
\end{array}\right.
$$

To initialise the iterative descent method we choose an initial datum $u^{0}$ given by

$$
u^{0}(x, y)=\left\{\begin{array}{cl}
0.4 & x \leq 0.3, \quad y \leq 0.3 \\
0 & \text { otherwise }
\end{array}\right.
$$

and the computational domain $(0,1) \times(0,1)$, with $\Delta x=\Delta y=\Delta t=1 / 400$.

In Figure 13 we plot $\log (J)$ with respect to the number of iterations, for both, the alternating descent method proposed in this article and the purely discrete approach.

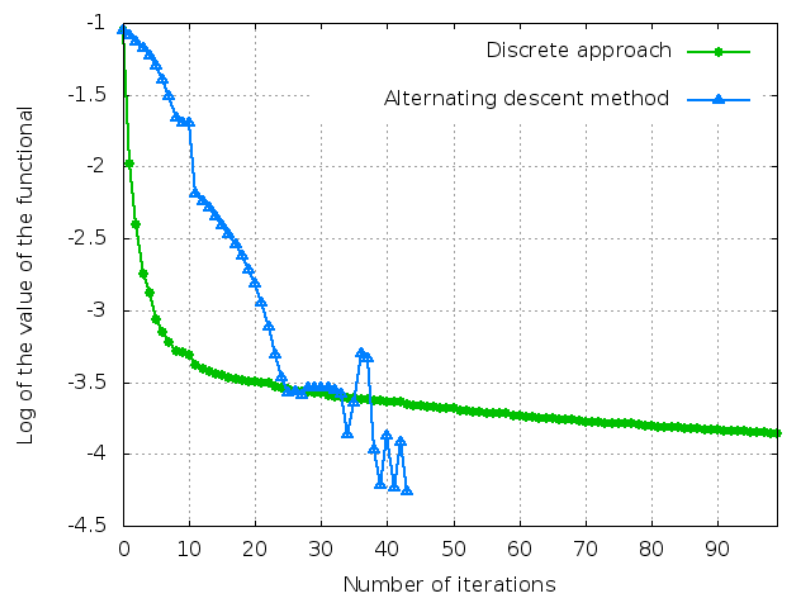

Figure 13. Experiment 2. Log of the value of the functional versus the number of iterations in the descent algorithm for the discrete approach scheme and the 2D alternating descent method.

In Figures 22-24, we illustrate the minimizers obtained by the methods above, and the associated solutions in time $T$.

\section{OPEN PROBLEMS}

We present some open problems and possible extensions of this work and the methods we have developed.

A significant number of issues arise even in the one-dimensional case. We mention here some of them: 
- Further develop and explore the efficiency of the alternated descent method for interacting shocks.

- Full implementation of the continuous approach developing a specific solver to approximate the adjoint system and compute numerical approximations of generalized gradients.

- Analysis of the efficiency of a purely discrete method in which the adjoint system is resolved in a coarser grid or, even, by a different more diffusive scheme as Lax-Friedfrichs, in opposition to the solver of the flow equation, taken to be, for instance Engquist-Osher, to better resolve the shocks.

- Adaptation of the alternating descent method to viscous equations with small viscosity, see [13] for preliminary work on this direction.

- Adaptation of the alternating descent method to systems of conservation laws.

In the multi-dimensional case, the 1D problems above can also be addressed, of course, but some other specific issues related to the multi-dimensional aspects also emerge. We mention here some of them.

- The problem of interacting shocks in the multi-dimensional setting could be of particular interest due to its geometric complexity.

- Take account of the finiteness of the multi-dimension shock and the added terms that its extremes or boundaries may lead to in the linearization of the functionals.

- Develop specific numerical methods for approximation the linearized equations and the adjoint system, paying special attention to the transport equation governing the sensivity of the shocks.

\section{FurThER NUMERICAL EXPERIMENTS}
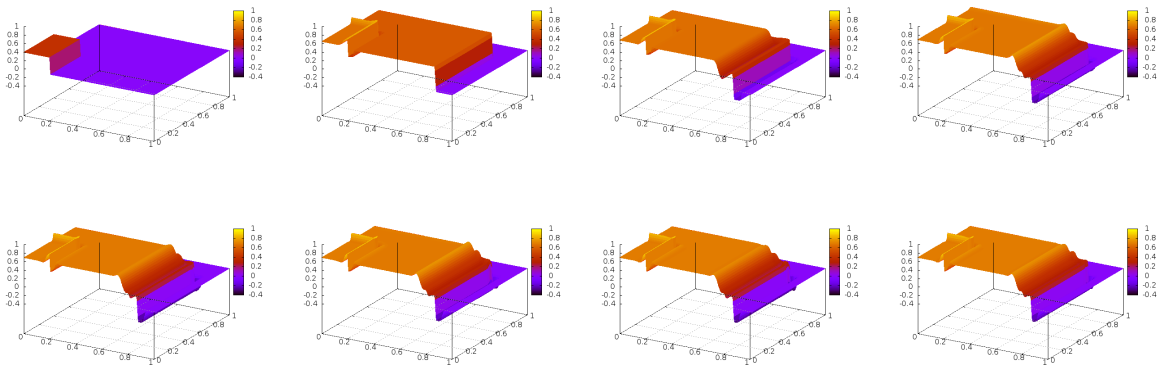

FIGURE 14. Iterations of the discrete approach in experiment 1. 

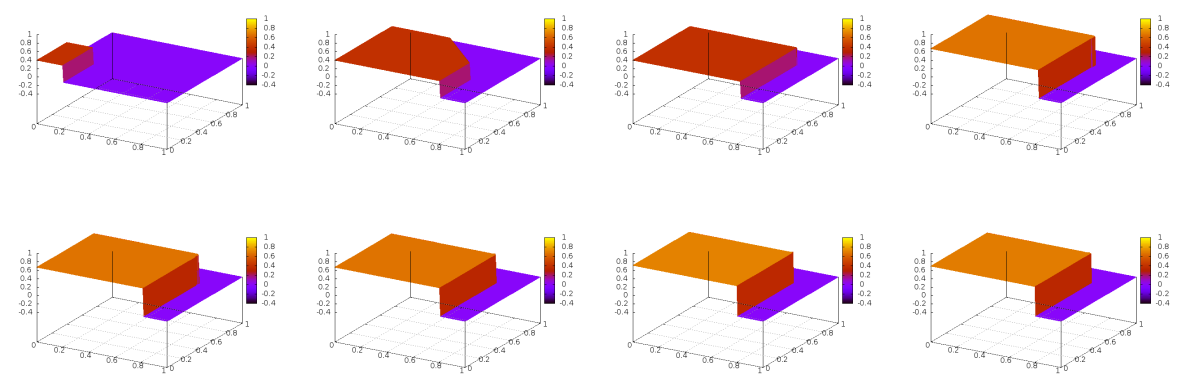

FIGURE 15. Iterations of the alternating descent method in experiment 1.

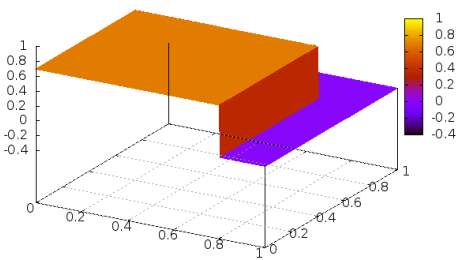

FIGURE $16 . \quad u^{0}$, alternating descent method, iteration $k=$ 56

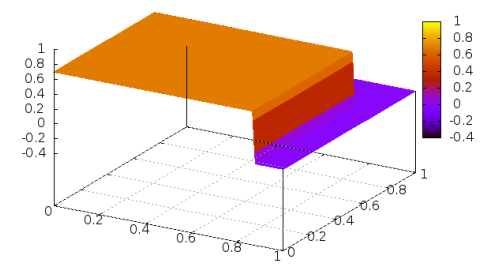

FiguRE 18. $u^{T}$, alternating descent method, iteration $k=$ 56

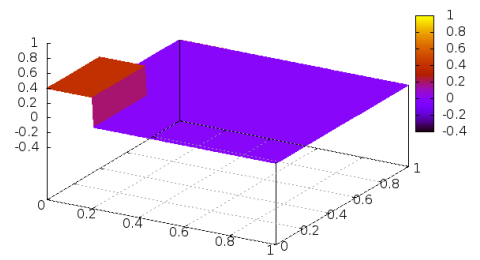

Figure 17. $u^{0}$, the discrete approach, iteration $k=99$

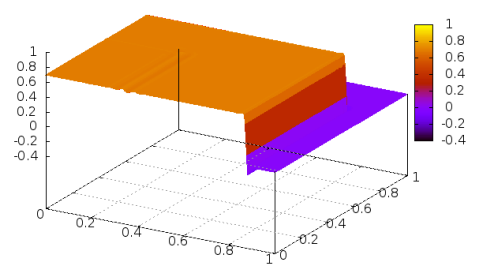

FiguRE 19. $u^{T}$, the discrete approach, iteration $k=99$ 


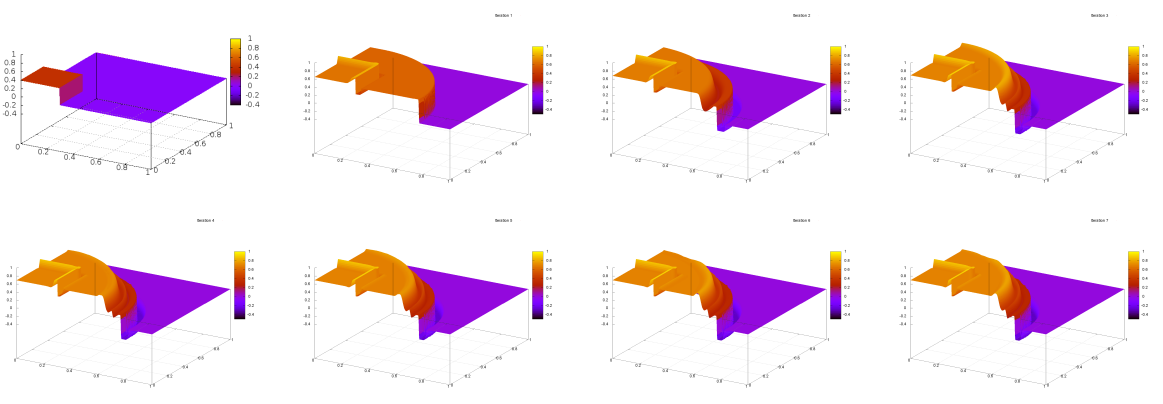

FiguRE 20. Iterations of the discrete approach in experiment 2.
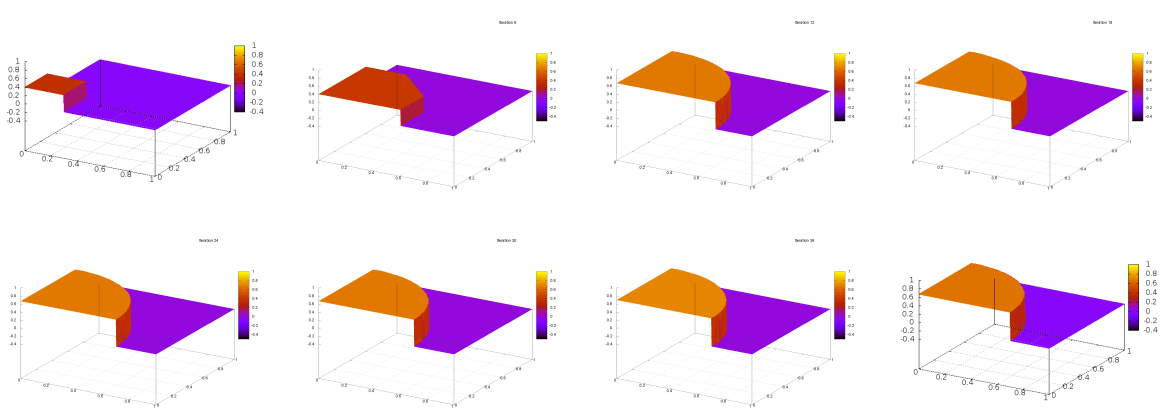

FiguRE 21. Iterations of the alternating descent method in experiment 2.

\section{Acknowledgements}

The authors would like to thank C. Castro, F. Palacios and A. Pozo for stimulation discussions.

\section{REFERENCES}

[1] Adimurthi, S. S. Ghoshal, and G. D. V. Gowda. Exact controllability of scalar conservation law with strict convex flux. http://hal .upmc.fr/docs/00/87/35/53/PDF/ExactControl .pdf., preprint, 2013.

[2] F. Ancona and G. M. Coclite. On the attainable set for temple class systems with boundary controls. SIAM J. Control Optim., 43(6):2166-2190, June 2005.

[3] F. Ancona and A. Marson. On the attainable set for scalar nonlinear conservation laws with boundary control. SIAM Journal on Control and Optimization, 36(1):290-312, 1998.

[4] D. Auroux and J. Blum. Back and forth nudging algorithm for data assimilation problems. Comptes Rendus Mathematique, 340(12):873 - 878, 2005.

[5] C. Bardos and O. Pironneau. A formalism for the differentiation of conservation laws. C. R. Math. Acad. Sci. Paris, 335(10):839-845, 2002.

[6] F. Bouchut and F. James. One-dimensional transport equations with discontinuous coefficients. Nonlinear Anal., 32(7):891-933, 1998.

[7] F. Bouchut and F. James. Differentiability with respect to initial data for a scalar conservation law. In Hyperbolic problems: theory, numerics, applications, Vol. I (Zürich, 1998), volume 129 of Internat. Ser. Numer. Math., pages 113-118. Birkhäuser, Basel, 1999.

[8] T. Boukadida and A. Y. Leroux. A new version of the two-dimensional lax-friedrichs scheme. Mathematics of Computation, 63(208):541-553, 1994. 


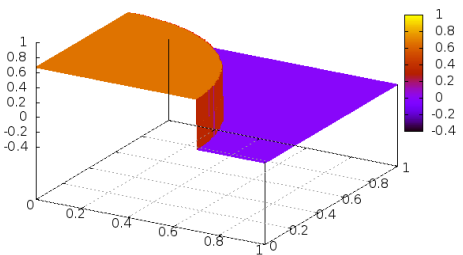

FIGURE $22 . \quad u^{0}$, alternating descent method, iteration $k=$

43

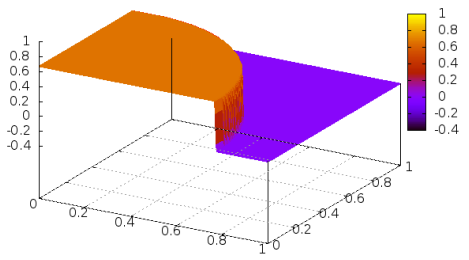

FIGURE 24. $u^{T}$, alternating descent method, iteration $k=$ 43

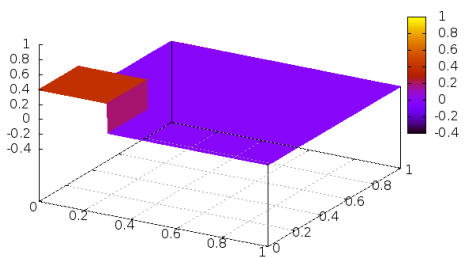

Figure 23. $u^{0}$, the discrete approach, iteration $k=99$

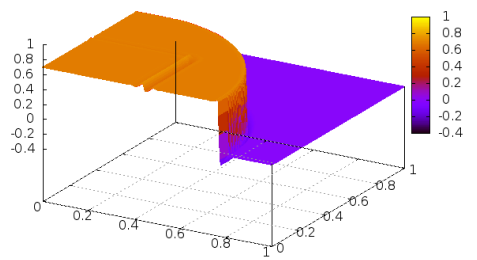

FIGURE 25. $u^{T}$, the discrete approach, iteration $k=99$

[9] A. Bressan and A. Marson. A maximum principle for optimally controlled systems of conservation laws. Rend. Sem. Mat. Univ. Padova, 94:79-94, 1995.

[10] A. Bressan and A. Marson. A variational calculus for discontinuous solutions of systems of conservation laws. Comm. Partial Differential Equations, 20(9-10):1491-1552, 1995.

[11] J. Canny. A computational approach to edge detection. Pattern Analysis and Machine Intelligence, IEEE Transactions on, PAMI-8(6):679-698, 1986.

[12] C. Castro, F. Palacios, and E. Zuazua. An alternating descent method for the optimal control of the inviscid burgers equation in the presence of shocks. Mathematical Models and Methods in Applied Sciences, 18(03):369-416, 2008.

[13] C. Castro, F. Palacios, and E. Zuazua. Optimal control and vanishing viscosity for the burgers equation. In C. Constanda and M. Pérez, editors, Integral Methods in Science and Engineering, Volume 2, pages 65-90. Birkhäuser Boston, 2010.

[14] C. Castro and E. Zuazua. Flux identification for 1-d scalar conservation laws in the presence of shocks. Math. Comp., 80(276):2025-2070, 2011.

[15] M. Crandall and A. Majda. The method of fractional steps for conservation laws. Numerische Mathematik, 34(3):285-314, 1980.

[16] M. Davis and M. David. An algorithm for finding the position of a point relative to a fixed polygonal boundary. Journal of the International Association for Mathematical Geology, 12(1):61-68, 1980.

[17] F. Dubois and P. LeFloch. Boundary conditions for nonlinear hyperbolic systems of conservation laws. J. Differential Equations, 71(1):93-122, 1988.

[18] L. Evans. Partial differential equations. Graduate studies in mathematics. American Mathematical Society, 1998. 
[19] C. Fabre, J.-P. Puel, and E. Zuazua. On the density of the range of the semigroup for semilinear heat equations. In J. Lagnese, D. Russell, and L. White, editors, Control and Optimal Design of Distributed Parameter Systems, volume 70 of The IMA Volumes in Mathematics and its Applications, pages 73-91. Springer New York, 1995.

[20] S. Garreau, P. Guillaume, and M. Masmoudi. The topological asymptotic for pde systems: The elasticity case. SIAM J. Control Optim., 39(6):1756-1778, Dec. 2000.

[21] B. Giles, Niles, and A. Pierce. Analytic adjoint solutions for the quasi-one-dimensional euler equations. J. Fluid Mechanics, pages 327-345, 2001.

[22] E. Godlewski and P. Raviart. Hyperbolic systems of conservation laws, Mathématiques and Applications. Ellipses, Paris, 1991.

[23] E. Godlewski and P. A. Raviart. The linearized stability of solutions of nonlinear hyperbolic systems of conservation laws. A general numerical approach. Math. Comput. Simulation, 50(1-4):77-95, 1999. Modelling '98 (Prague).

[24] L. Gosse. A two-dimensional version of the godunov scheme for scalar balance laws. SIAM Journal on Numerical Analysis, 52(2):626-652, 2014.

[25] L. Gosse and F. James. Numerical approximations of one-dimensional linear conservation equations with discontinuous coefficients. Mathematics of Computation, 69(231):pp. 987$1015,2000$.

[26] J. Hall. Ptloc-a fortran subroutine for determining the position of a point relative to a closed boundary. Journal of the International Association for Mathematical Geology, 7(1):75-79, 1975.

[27] F. James and M. Sepúlveda. Convergence results for the flux identification in a scalar conservation law. SIAM Journal on Control and Optimization, 37(3):869-891, 1999.

[28] S. N. Kružkov. First order quasilinear equations with several independent variables. Mat. Sb. (N.S.), 81 (123):228-255, 1970.

[29] A. Majda. The stability of multidimensional shock fronts. Mem. Amer. Math. Soc., 41(275):iv+95, 1983.

[30] D. Mumford and J. Shah. Optimal approximations by piecewise smooth functions and associated variational problems. Communications on Pure and Applied Mathematics, 42(5):577685, 1989.

[31] L. I. Rudin, S. Osher, and E. Fatemi. Nonlinear total variation based noise removal algorithms. Phys. D, 60(1-4):259-268, Nov. 1992.

[32] J. Sethian. Level Set Methods and Fast Marching Methods: Evolving Interfaces in Computational Geometry, Fluid Mechanics, Computer Vision, and Materials Science. Cambridge Monographs on Applied and Computational Mathematics. Cambridge University Press, 1999.

[33] S. Ulbrich. Adjoint-based derivative computations for the optimal control of discontinuous solutions of hyperbolic conservation laws. Systems Control Lett., 48(3-4):313-328, 2003. Optimization and control of distributed systems.

[34] X. Wen and S. Jin. Convergence of an immersed interface upwind scheme for linear advection equations with piecewise constant coefficients. I. $L^{1}$-error estimates. J. Comput. Math., 26(1):1-22, 2008.

[35] Y. Zheng. Systems of Conservation Laws: Two-Dimensional Riemannian Problems. Progress in Nonlinear Differential Equations and Their Applications. Birkhäuser Boston, 2001.

Rodrigo Lecaros ${ }^{1,3}$ And Enrique ZuazuA ${ }^{1,2}$

${ }^{1}$ BCAM - Basque Center for Applied Mathematics,

Mazarredo 14, E-48009, Bilbao, Basque Country, Spain

${ }^{2}$ Ikerbasque - Basque Foundation for Science,

Alameda Urquijo 36-5, Plaza Bizkaia, 48011, Bilbao, Basque Country, Spain

${ }^{3}$ CMM - Centro de Modelamiento Matemático. Universidad de Chile (UMi CNRS 2807),

Avenida Blanco Encalada 2120, Casilla 170-3, Correo 3, Santiago, Chile

E-mail address: rlecaros@dim.uchile.cl,zuazua@bcamath.org

$U R L$ : www.bcamath.org/zuazua/ 